\title{
Three-Valued Logics, Uncertainty Management and Rough Sets ${ }^{\star}$
}

\author{
Davide Ciucci $^{1}$ and Didier Dubois ${ }^{2}$ \\ 1 DISCo - Università di Milano - Bicocca \\ Viale Sarca 336 - U14, 20126 Milano (Italia) \\ 2 IRIT, Université Paul Sabatier \\ 118 route de Narbonne, 31062 Toulouse cedex 4 (France)
}

\begin{abstract}
This paper is a survey of the connections between threevalued logics and rough sets from the point of view of incomplete information management. Based on the fact that many three-valued logics can be put under a unique algebraic umbrella, we show how to translate three-valued conjunctions and implications into operations on ill-known sets such as rough sets. We then show that while such translations may provide mathematically elegant algebraic settings for rough sets, the interpretability of these connectives in terms of an original set approximated via an equivalence relation is very limited, thus casting doubts on the practical relevance of truth-functional logical renderings of rough sets.
\end{abstract}

\section{Introduction}

Rough sets have often been studied under a three-valued logic framework and different authors have tried to connect rough sets to different logics: Łukasiewicz $[9,11]$, Nelson [58, 59], Gödel, Gaines-Rescher three-valued logics [49, 41]. Despite the formal correctness of these approaches, little attention has been devoted to the interpretation of these logics in the rough set context. Moreover, a comprehensive study on the three-valued connectives that can be defined on rough sets is needed and, as we will see, it can be accomplished starting from known results in three-valued logics.

Three-valued logics are apparently simple; they are straightforward generalizations of Boolean logic based on the most simple bipolar scale $\left\{0, \frac{1}{2}, 1\right\}$ where 1 (resp. 0) has a positive (resp. negative) flavor, and $\frac{1}{2}$ is neutral. Further, they are widely used in several applied contexts such as logic programming [43], electronic circuits [67], databases [27], and, of course, rough sets. However, there have been several different meanings attached to the third value, some having an epistemic nature. There is not a clear result on the definition of its connectives in connection with this meaning. Here is a list of these interpretations of the third truth-value, different from true and false : Possible (due to Eukasiewicz

\footnotetext{
* Appeared in Transactions on Rough Sets XVII, Lecture Notes in Computer Science Volume 8375, 2014, pp 1-32, DOI: 10.1007/978-3-642-54756-0_1
} 
[17]), Unknown (Kleene [52]), Undefined (also Kleene), Half-true (in fuzzy logic [48]), Borderline (in logics of vagueness, like in Shapiro [66]), Inconsistent (that is both true and false, as in paraconsistent logics or the logic of paradox by Priest [63]), or yet Irrelevant as in relevance logics [2] or the logic of conditionals [38]. Sometimes, two of these notions are simultaneously used as Inconsistent and Unknown in Belnap four-valued logic [14].

Three-valued logics go along with three-valued sets having central elements and peripheral ones [46]. However the meaning of such central and peripheral elements depends on the meaning of the third truth-value. It depends on whether it has an epistemic flavor or not; a peripheral element can be understood in one of the following ways:

1. either as an untypical element of a non-classical set,

2. or as an element that cannot be definitely classified as belonging or not to a crisp set due to incomplete information,

3. or as an element that cannot be definitely classified as belonging or not to a crisp set due to conflicting information,

4. or as an element for which membership or non membership makes no sense, due to irrelevance or the dubious existence of such an element.

Case 2 is the one we are concerned with in this paper. Then the three truthvalues refer to the epistemic status of otherwise Boolean propositions (provably true, provably false or unknown [39]). This is typically the case of ill-known or interval sets [72], where the central elements are elements that certainly belong to some ill-known set, the third truth-value is assimilated to $\{0,1\}$ and understood as the hesitancy between membership and non-membership. They are special cases of interval-valued fuzzy sets [77] or twofold fuzzy sets [37]. One of the causes of a set being ill-known can be the lack of precision on the value of some of the attributes that describe it (for instance, a set of single persons is ill-known if the marital status of some of the persons is ill-known).

A rough set, viewed as a pair of nested approximations is a typical example of ill-known set, where the lack of knowledge comes from an equivalence relation between possibly indistinguishable elements, this indistinguishability being due to the use of a language that is not expressive enough (incomplete set of attributes or attributes that are too coarsely defined). This situation contrasts with the case of sets that are ill-known due to the lack of knowledge of attribute values; see Couso and Dubois [28] when the two causes of partial ignorance appear simultaneously.

In recent papers [23-26], we have studied various three-valued logics of partial knowledge, where the third truth-value means unknown. It has been shown that a large class of three-valued logics (including Eukasiewicz $L_{3}$ ) is compatible with this understanding of the third truth-value, but their translations into a very elementary modal logic indicate that such three-valued logics cannot account for partial ignorance jointly affecting several Boolean variables: only states of partial ignorance that can be described independently for each variable can be accounted for in a three-valued logic. This is the price paid for truth-functionality. 
In this paper, we examine the situation of three-valued logics of rough sets. While the aforementioned limitation is still valid (since rough sets do not behave truth-functionally in general), there is an additional constraint in this case. Namely, the approximation pairs are generated by an equivalence relation, which create additional interpretive difficulties for truth-functional definitions of conjunction and disjunction [22]. In this paper, we consider the situation of more general three-valued connectives in connection with rough sets.

In the following, we review some results on three-valued logics, in particular we give a list of reasonable connectives on three values that can apply to ill-known sets. Then, these connectives are translated into the language of nested pairs or orthopairs of sets, showing that, from a formal point of view, this translation is correct. On the other hand, some considerations from the interpretation standpoint are put forward casting some doubts on this truth-functional approach to ill-known sets and rough sets. Especially, in the case of rough sets, it seems impossible to interpret combinations of rough sets in terms of pure combinations of the underlying ill-known sets (approximated via an equivalence relation). Finally, we discuss some modal and three-valued logics of rough sets in connection with a recent translation of three-valued logics into a fragment of the KD logic.

\section{Aggregation functions on three valued logics}

We denote by 3 the set $\left\{0, \frac{1}{2}, 1\right\}$ with the usual order: $0<\frac{1}{2}<1$. Due to the total order assumption, we can define the idempotent and commutative Kleene conjunction and disjunction, that is, the minimum, denoted by $\sqcap$ and the maximum denoted by $\sqcup: x \sqcap y=y \sqcap x=x$ if and only if $x \leq y$ if and only if $x \sqcup y=y \sqcup x=y$. Moreover, Gödel implication is definable by residuation:

$$
x \sqcap y \leq z \text { if and only if } x \leq y \rightarrow_{G} z .
$$

It is such that $y \rightarrow_{G} z=1$ if $y \leq z$ and $z$ otherwise. Finally, the intuitionistic negation is obtainable by Gödel implication as $\sim x=x \rightarrow_{G} 0$. We now report some results [25] about three-valued logics: a list of possible connectives, the logical systems they generate and the links among them.

\subsection{Connectives}

A maximal family of sensible conjunctions and implications on $\mathbf{3}$ is now recalled, based on some intuitive properties, in the scope of modeling incomplete information. Then, negation and disjunction can be derived respectively as $a \rightarrow 0$ and by De Morgan properties.

Definition 2.1. A conjunction on $\mathbf{3}$ is a binary mapping $*: \mathbf{3} \times \mathbf{3} \mapsto \mathbf{3}$ that is monotonically increasing in the wide sense, and extends the connective AND in Boolean logic:

(C1) If $x \leq y$ then $x * z \leq y * z$; 
(C2) If $x \leq y$ then $z * x \leq z * y$;

(C3) $0 * 0=0 * 1=1 * 0=0$ and $1 * 1=1$.

Bearing in mind our focus on the epistemic understanding of the third truthvalue as unknown, condition C3 is clearly natural if we notice that, in consequence to this interpretive assumption, 1 must mean "certainly true" and 0 "certainly false", which justifies this requirement of coincidence with Boolean conjunction for truth-values different from $1 / 2$.

Due to (C3), the monotonicity properties (C1-C2) imply $\frac{1}{2} * 0=0 * \frac{1}{2}=0$. It goes along with the fact that a conjunction is false whenever one of the conjuncts is false, regardless of whether the truth-value of the other conjunct is known or not. If we consider all the possible cases, there are 14 conjunctions satisfying definition 2.1. Among them, only six are commutative and only five associative. These five conjunctions are already known in the literature and precisely, they have been studied in the following logics: Sette [65], Sobociński [68], Eukasiewicz [17], Kleene [52], Bochvar [15]. The complete list is given in Table 1.

\begin{tabular}{|c|c|c|c|c|c|c|c|}
\hline & & & & $*$ & & & \\
\hline & & & \begin{tabular}{l|l}
1 \\
\end{tabular} & 1 & 1 & 1 & Sette \\
\hline & & & 2 & $\frac{1}{2}$ & 1 & 1 & quasi conjunction/Sobociński \\
\hline & & & \begin{tabular}{l|l}
3 & \\
\end{tabular} & $\frac{1}{2}$ & 1 & $\frac{1}{2}$ & \\
\hline & & & 4 & $\frac{1}{2}$ & $\frac{1}{2}$ & 1 & \\
\hline$* \mid 0$ & $\frac{1}{2}$ & 1 & 5 & $\frac{1}{2}$ & $\frac{1}{2}$ & $\frac{1}{2}$ & min/interval conjunction/Kleene \\
\hline \begin{tabular}{l|l}
0 & 0
\end{tabular} & 0 & 0 & 6 & 0 & 0 & 1 & \\
\hline \begin{tabular}{l|l}
$\frac{1}{2}$ & 0
\end{tabular} & & & 7 & 0 & 0 & $\frac{1}{2}$ & \\
\hline \begin{tabular}{l|l}
2 \\
1 & 0
\end{tabular} & & 1 & 8 & 0 & 0 & 0 & Bochvar external \\
\hline & & & 9 & 0 & $\frac{1}{2}$ & 0 & \\
\hline & & & $\overline{10}$ & 0 & $\frac{1}{2}$ & 1 & \\
\hline & & & 11 & 0 & $\frac{1}{2}$ & $\frac{1}{2}$ & Łukasiewicz \\
\hline & & & $\overline{12}$ & 0 & $\frac{2}{1}$ & 0 & \\
\hline & & & 13 & 0 & 1 & $\frac{1}{2}$ & \\
\hline & & & $\overline{14}$ & 0 & 1 & 1 & \\
\hline
\end{tabular}

Table 1. All conjunctions on $\mathbf{3}$ according to Definition 2.1

Besides Definition 2.1 other possible definitions of conjunction can be found in literature:

- (conjunction of conditional events) due to Walker [71]. The required properties are the coincidence with Boolean conjunction on Boolean values $\{0,1\}$, idempotence and commutativity. Only nine conjunctions satisfy these axioms, among them Sobociński's (also Adams quasi-conjunction of conditionals [1]) and the two Kleene ones. The other six are all non-monotonic and only one is associative. Moreover, three of them are such that $\frac{1}{2} * 0=1$. All 
these facts cast some doubts on the interpretability of these six conjunctions on 3 outside the setting of conditional events.

- t-norms, uninorms $[54,29]$. A uninorm is a binary operator which is associative, commutative, non-decreasing in each component and with a neutral element $e: \forall x, e * x=x$. A t-norm is a uninorm such that $e=1$. Among conjunctions on $\mathbf{3}$ we have only two t-norms: Gödel and Lukasiewicz and only one more uninorm: Sobociński. They already appear in the above Table 1.

- t-operators [54]: an associative, commutative binary operators such that $0 *$ $0=0,1 * 1=1$ and satisfying 1-smoothness: $x_{i} * x_{j-1} \leq x_{i} * x_{j}$ and if $x_{i} * x_{j}=$ $x_{k}$ then $\left\{x_{i-1} * x_{j}, x_{i} * x_{j-1}\right\} \subseteq\left\{x_{k}, x_{k-1}\right\}$. Besides Kleene and Lukasiewicz conjunctions and disjunctions, on three values we get one more operator: the median $\operatorname{med}\left(x, y, \frac{1}{2}\right)$, which, however, does not generalize Boolean logic.

In the case of implication, we can give a general definition, which extends Boolean logic and supposes monotonicity (decreasing in the first argument, increasing in the second).

Definition 2.2. An implication on $\mathbf{3}$ is a binary mapping $\rightarrow: \mathbf{3} \times \mathbf{3} \mapsto \mathbf{3}$ such that

(I1) If $x \leq y$ then $y \rightarrow z \leq x \rightarrow z$;

(I2) If $x \leq y$ then $z \rightarrow x \leq z \rightarrow y$;

(I3) $0 \rightarrow 0=1 \rightarrow 1=1$ and $1 \rightarrow 0=0$.

From the above definition we derive $x \rightarrow 1=1,0 \rightarrow 1=1$ and $\frac{1}{2} \rightarrow \frac{1}{2} \geq$ $\left\{1 \rightarrow \frac{1}{2}, \frac{1}{2} \rightarrow 0\right\}$. There are 14 implications satisfying this definition, listed in Table 2 .

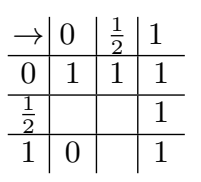

\begin{tabular}{c|c|c|c|c|}
$\mathrm{n}$. & $\frac{1}{2} \rightarrow \frac{1}{2}$ & $1 \rightarrow \frac{1}{2}$ & $\frac{1}{2} \rightarrow 0$ & \\
\hline \hline 1 & 0 & 0 & 0 & \\
\hline \hline 2 & $\frac{1}{2}$ & 0 & 0 & Sobociński \\
\hline 3 & $\frac{1}{2}$ & 0 & $\frac{1}{2}$ & \\
\hline 4 & $\frac{1}{2}$ & $\frac{1}{2}$ & 0 & Jaśkowski \\
\hline 5 & $\frac{1}{2}$ & $\frac{1}{2}$ & $\frac{1}{2}$ & (strong) Kleene \\
\hline \hline 6 & 1 & 1 & 0 & Sette \\
\hline 7 & 1 & 1 & $\frac{1}{2}$ & \\
\hline 8 & 1 & 1 & 1 & \\
\hline 9 & 1 & $\frac{1}{2}$ & 1 & Nelson \\
\hline 10 & 1 & $\frac{1}{2}$ & 0 & Gödel \\
\hline 11 & 1 & $\frac{1}{2}$ & $\frac{1}{2}$ & Łukasiewicz \\
\hline 12 & 1 & 0 & 1 & Bochvar external \\
\hline 13 & 1 & 0 & $\frac{1}{2}$ & \\
\hline 14 & 1 & 0 & 0 & Gaines-Rescher
\end{tabular}

Table 2. All implications according to Definition 2.2 
Nine of them are known and have been studied. Besides those implications named after the five logics mentioned above, there are also those named after Jaśkowski [50], Gödel [47], Nelson [55], Gaines-Rescher [44].

Finally, there are only three possible negations that extend the Boolean negation, namely, if $0^{\prime}=1$ and $1^{\prime}=0$ :

1. $\sim \frac{1}{2}=0$. It corresponds to an intuitionistic negation, since it satisfies the law of contradiction, and not the excluded middle. It is of the form $a \rightarrow_{i} 0$ for implications $1,2,4,6,10,14$.

2. $\neg \frac{1}{2}=\frac{1}{2}$. It is an involutive negation. It is of the form $a \rightarrow_{i} 0$ for implications $3,5,7,11,13$.

3. $-\frac{1}{2}=1$. It is called a paraconsistent negation, since it satisfies the law of excluded middle, and not the one of contradiction. It is of the form $a \rightarrow_{i} 0$ for implications $8,9,12$.

\subsection{Logical systems}

As mentioned, some of these connectives have already been studied and they are at the basis of known logical formalisms. Here is a (possibly not exhaustive) list:

- Eukasiewicz logic $\left(\rightarrow_{11}, *_{11},+_{11}, \neg\right)$, where the disjunction $+_{11}$ definable by de Morgan properties as $a+{ }_{11} b:=\neg\left(\neg a *_{11} \neg b\right)$ is the truncated sum. We also recall that the interpretation given by Łukasiewicz for the third value is possible whereas, nowadays, Łukasiewicz logic is mainly used in many valued logics where the third value as a gradual truth meaning.

- Sobociński logic $\left(\rightarrow_{2}, *_{2},+_{2}, \neg\right)$ where $+_{2}$ can be defined as $a+{ }_{2} b:=\neg a \rightarrow_{2} b$ and designated values are $1, \frac{1}{2}$. In this case, the third value means irrelevant and it has been used in the context of relevance logics [2] and conditional events [38]. We recall that conjunction $*_{2}$ is a discrete uninorm with $\frac{1}{2}$ as neutral element and implication $\rightarrow_{2}$ its residuum [7].

- Gödel (intuitionistic) logic $\left(\rightarrow_{10}, *_{5}(\min ), \max , \sim\right)$ on three values, also known as logic of here-and-there in logic programming [62].

- Jaśkowski logic $\left(\rightarrow_{4}, *_{5}(\min )\right.$, max, $\left.\neg\right)$ has been studied by several authors in the field of paraconsistent logic $[32,2,4]$. The designated values are $\frac{1}{2}$ and 1 and the interpretation of the third value means inconsistent, paradoxical, that is, both true and false.

- Bochvar logic $\left(\rightarrow_{12}, *_{8},+_{8}, b\right)$ where $x+_{8} y$ is 1 if at least one of $a$ and $b$ is equal to 1 and 0 in all other cases. Third value $\frac{1}{2}$ stands for meaningless.

- Sette paraconsistent logic $\left(\rightarrow_{6}, *_{1},-\right)$ where $x+{ }_{1} y$ takes the value 0 if $x=$ $y=0$ and 1 otherwise and designated values are $\frac{1}{2}$ and 1 . We note that Sette conjunction (n.1) and implication (n.6) correspond to the collapse of the truth-values 1 and $\frac{1}{2}$. The author does not give a clear semantic to the third value and he introduces the logic as a "not absolutely inconsistent" formal system.

- Nelson logic $\left(\rightarrow_{9}, *_{5}, \max , \neg,-\right)$ where - is a paraconsistent negation and $\rightarrow_{9}$ Nelson implication. It is the logic of constructible falsity, and in this sense it is dual to intuitionistic logic. On five values it is also known as equilibrium logic in the context of logic programming [62]. 
The reader is referred to the work of Avron [5] for a reconstruction of some of the above three-valued logics (said to be "natural") where the third truth-value is understood as unknown or contradictory, based on the inferential standpoint.

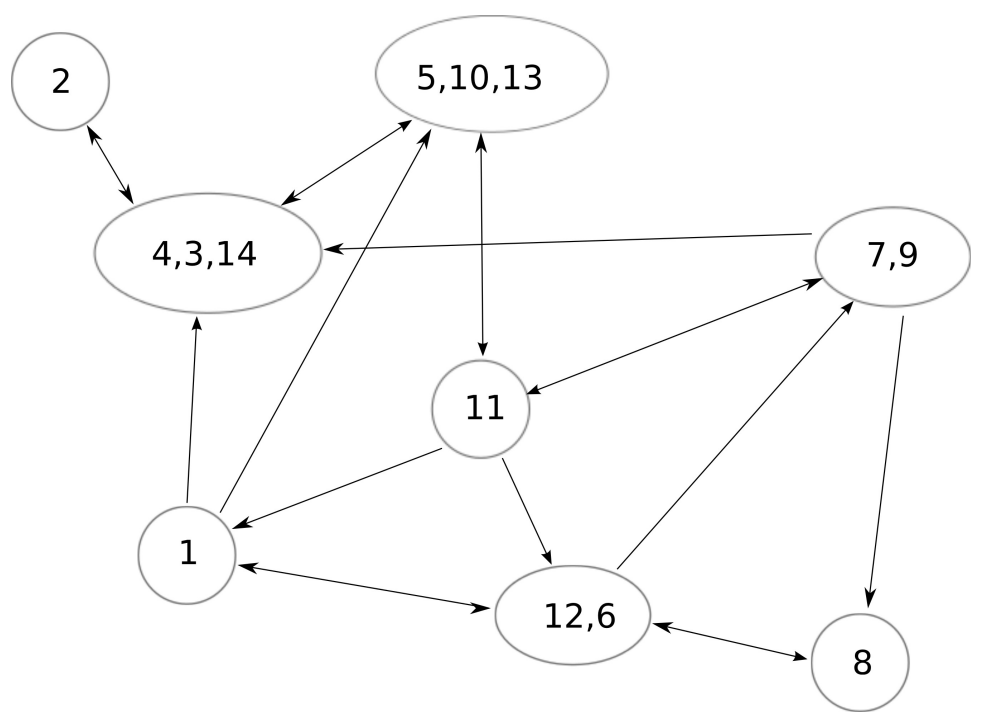

Fig. 1. Outline of all the relations among connectives.

\subsection{Connections among logics}

Some relations among the above systems are known. For instance, Sette logic has been obtained in [32] from Łukasiewicz logic in order to demonstrate a relationship between many-valued and paraconsistent logics; likewise, it can be proved that Jaśkowski and Sobociński logics are equivalent [5]. However, other connections can be put forward by a systematic study. As a result we can prove that all these systems and more generally, all the 14 conjunctions and implications are inter-definable. More precisely, we consider the following transformations of a binary operator $\odot$ on $\mathbf{3}[35,36]$ :

$$
\begin{aligned}
& a[\mathcal{A}(\odot)] b=b \odot a \\
& a[\mathcal{V}(\odot)] b=\neg b \odot \neg a \\
& a[\mathcal{S}(\odot)] b=\neg(a \odot \neg b) \\
& a[\mathcal{I}(\odot)] b=\left\{\begin{array}{ll}
0 & \nexists s, a \odot s \leq b ; \\
\sup \{s: a \odot s \leq b\}, & \text { otherwise. }
\end{array}\right. \text { (material implication) (1c) }
\end{aligned}
$$


We also define relations among implications through some formulae which are tautologies in Boolean logic:

$$
\begin{aligned}
& a \rightarrow_{\text {new }} b=(a \rightarrow b) \wedge(\neg b \rightarrow \neg a) ; \\
& a \rightarrow_{\text {new }} b=b \vee(a \rightarrow b) ; \\
& a \rightarrow_{\text {new }} b=a \rightarrow(a \rightarrow b) ; \\
& a \rightarrow_{\text {new }} b=(a \rightarrow b) \vee(\neg b \rightarrow \neg a) ; \\
& a \rightarrow_{\text {new }} b=\neg a \vee(a \rightarrow b)
\end{aligned}
$$

In the graph of Figure 1, a representation of all these relationships is given: every circle represents a group of conjunctions/implications related via transformations defined in equations (1), whereas groups are linked by transformations defined in equations (2). These transformations are instrumental to get the following results $[23,25]$ :

Proposition 2.1. Let 3 be the three-element set with the usual order $0<\frac{1}{2}<1$ or equivalently, $\mathbf{3}=(\overline{3}, \wedge, \vee)$, with $\overline{3}$ the set of three elements without the order structure. All the 14 conjunctions and implications can be defined in any of the following systems:

- $\left(\mathbf{3}, \neg, \rightarrow_{10}\right)=\left(\overline{3}, \wedge, \neg, \rightarrow_{10}\right)$ (Gödel implication plus the involutive negation);

- $\left(\mathbf{3}, \rightarrow_{i}\right)=\left(\overline{3}, \wedge, \vee, \rightarrow_{i}\right)$ where $i \in I=\{3,5,7,11,13\}$, allowing residuation.

Further, we can also consider a set with three elements without a predefined order (and so without min, max and residuation) and obtain the following proposition.

Proposition 2.2. We denote by $\overline{3}$ the set of three elements without any structure. All the 14 conjunctions and implications can be defined in any of the following systems:

- $\left(\overline{3}, \rightarrow_{11}, 0\right)$ where $\rightarrow_{11}$ is Lukasiewicz implication;

- $\left(\overline{3}, \rightarrow_{9}, \neg\right)$ where $\rightarrow_{9}$ is Nelson implication;

- $\left(\overline{3}, \rightarrow_{5}, \sim, 0\right)$ where $\rightarrow_{5}$ is Kleene implication and $\sim$ the intuitionistic negation.

- $\left(\overline{3}, \rightarrow_{5},-, 0\right)$ where $\rightarrow_{5}$ is Kleene implication and - the paraconsistent negation.

In the two arrays of Table 3 we report how to obtain all the conjunctions and implications starting from the Eukasiewicz implication $\rightarrow_{L}=\rightarrow_{11}$ and 0 ; of course $\neg a=a \rightarrow_{L} 0$. We denote by $\odot$ Eukasiewicz conjunction; moreover, $\nabla a$ is an abbreviation for $\neg a \rightarrow_{L} a, \Delta(a)$ stands for $a \odot a=\neg\left(a \rightarrow_{L} \neg a\right)$, and finally $J(a)$ is short for $\neg a *_{1} \neg\left(\neg a *_{1} a\right)=\nabla(\neg a \wedge \neg \nabla(\neg a \wedge a))$. Clearly $\sim a=\neg \nabla a$ and $-a=\neg \Delta a$.

So, the differences among three-valued logics are just apparent. All of them can be interpreted as a fragment of the same logic, such as Łukasiewicz logic, or sometimes a variant thereof with the same expressive power (like Nelson's logic). According to the purpose and to the desired interpretation, we can then choose the proper fragment and connectives. 


\begin{tabular}{c|c|c|}
$n$ & $a *_{n} b$ & \\
\hline \hline 1 & $\nabla(a \wedge b)$ & \\
\hline 2 & $\nabla(a \wedge b) \wedge(a \vee b)$ & Sobociński \\
\hline 3 & $\nabla(a \wedge b) \wedge a$ & \\
\hline 4 & $\nabla(a \wedge b) \wedge b$ & \\
\hline 5 & $a \wedge b$ & (strong) Kleene \\
\hline 6 & $\neg J(a) \odot J(\neg b)$ & \\
\hline 7 & $a \odot(\neg b \odot a)$ & \\
\hline 8 & {$[\neg b \odot(\neg b \odot a)] \wedge[\neg a \odot(\neg a \odot \neg b)]$} & Bochvar external \\
\hline 9 & $a \odot b=\neg\left(\neg a \rightarrow \rightarrow_{L} b\right)$ & \\
\hline 10 & $b \wedge[(\neg J(a) \odot J(\neg b)) \vee(J(\neg a) \odot \neg J(b))]$ & \\
\hline 11 & $J(\neg a) \odot \neg J(b)$ & \\
\hline 12 & $a \wedge[(\neg J(a) \odot J(\neg b)) \vee(J(\neg a) \odot \neg J(b))]$ & \\
\hline 13 & $a \wedge(\neg(a) \odot J(\neg b)) \vee(J(\neg a) \odot \neg J(b))$ & \\
\hline 14 & $(\neg J(a)$ &
\end{tabular}

\begin{tabular}{c|c|c|}
$n$ & $a \rightarrow_{n} b$ & \\
\hline \hline 1 & $\Delta(\neg a) \vee \Delta(b)$ & \\
\hline 2 & $\left(b \vee\left(a \rightarrow_{1} b\right)\right) \wedge\left(\neg a \vee\left(\neg b \rightarrow_{1} \neg a\right)\right)$ & Sobociński \\
\hline 3 & $\neg a \vee\left[\left(b \vee\left(a \rightarrow_{1} b\right) \wedge\left(\neg a \vee\left(\neg b \rightarrow_{1} \neg a\right)\right)\right]\right.$ & \\
\hline 4 & $b \vee(\Delta(\neg a) \vee \Delta(b))$ & Jaśkowski \\
\hline 5 & $\neg a \vee(\Delta(\neg a) \vee \Delta(b))$ & (strong) Kleene \\
\hline 6 & $J(b) \rightarrow_{L} J(a)$ & Sette \\
\hline 7 & $\neg b \rightarrow_{L}\left(\neg b \rightarrow_{L} \neg a\right)$ & Nelson \\
\hline 8 & $\left.a \rightarrow_{L}\left(a \rightarrow_{L} b\right)\right) \vee\left(\neg b \rightarrow_{L}\left(\neg b \rightarrow_{L} \neg a\right)\right)$ & Gödel \\
\hline 9 & $a \rightarrow_{L}\left(a \rightarrow_{L} b\right)$ & Bochvar external \\
\hline 10 & $\left.\neg_{L}\left(\left(\alpha \rightarrow_{L} \beta\right) \rightarrow_{L} \beta\right)\right)$ & \\
\hline 12 & $J(\neg a) \rightarrow_{L} J(\neg b)$ & \\
\hline 13 & $\neg a \vee\left[\left(J(\neg a) \rightarrow_{L} J(\neg b)\right) \wedge\left(J(b) \rightarrow_{L} J(a)\right)\right]$ & \\
\hline 14 & $\left(J(\neg a) \rightarrow_{L} J(\neg b)\right) \wedge\left(J(b) \rightarrow_{L} J(a)\right)$ & Gaines-Rescher
\end{tabular}

Table 3. All connectives expressed using Łukasiewicz logic operators.

\subsection{Connectives on nested pairs and orthopairs of sets}

Let $f: X \mapsto \mathbf{3}$ be a three-valued function that may be viewed as a special kind of fuzzy set. Then, from each $f$, we can induce three (Boolean) subsets forming a partition of the universe $X$ :

$$
\begin{array}{rr}
A_{1}:=\{x: f(x)=1\} & \text { The truth domain; } \\
A_{0}:=\{x: f(x)=0\} & \text { The falsity domain; } \\
A_{\frac{1}{2}}:=\left\{x: f(x)=\frac{1}{2}\right\} & \text { The neutral domain. }
\end{array}
$$

Formally, we can see any three-valued set $f$ as a pair $\left(A_{1}, A_{0}\right)$ of classical sets satisfying the property $A_{1} \cap A_{0}=\emptyset$, i.e., $A_{1}$ and $A_{0}$ are disjoint sets and $\left(A_{1}, A_{0}\right)$ is called an orthopair [21]. Conversely, given a pair of disjoint sets, we 


\begin{tabular}{c|c}
$\mathrm{n}$ & $\left(A_{1}, A_{0}\right) *\left(B_{1}, B_{0}\right)$ \\
\hline 1 & $\left(A_{0}^{c} \cap B_{0}^{c}, A_{0} \cup B_{0}\right)$ \\
\hline 2 & $\left(\left(A_{1} \cup B_{1} \cap \cap A_{0}^{c} \cap B_{0}^{c}, A_{0} \cup B_{0}\right)\right.$ \\
\hline 3 & $\left(A_{1} \cap B_{0}^{c}, A_{0} \cup B_{0}\right)$ \\
\hline 4 & $\left(A_{0}^{c} \cap B_{1}, A_{0} \cup B_{0}\right)$ \\
\hline 5 & $\left(A_{1} \cap B_{1}, A_{0} \cup B_{0}\right)$ \\
\hline 6 & $\left(A_{0}^{c} \cap B_{1}, A_{0} \cup B_{1}^{c}\right)$ \\
\hline 7 & $\left(A_{1} \cap B_{1}, A_{0} \cup B_{1}^{c}\right)$ \\
\hline 8 & $\left(A_{1} \cap B_{1}, A_{1}^{c} \cup B_{1}^{c}\right)$ \\
\hline 9 & $\left(A_{1} \cap B_{1}, A_{1}^{c} \cup B_{0}\right)$ \\
\hline 10 & $\left(A_{0}^{c} \cap B_{1},\left(A_{1}^{c} \cap B_{1}^{c}\right) \cup A_{0} \cup B_{0}\right)$ \\
\hline 11 & $\left(A_{1} \cap B_{1},\left(A_{1}^{c} \cap B_{1}^{c}\right) \cup A_{0} \cup B_{0}\right)$ \\
\hline 12 & $\left(A_{1} \cap B_{0}^{c}, A_{1}^{c} \cup B_{0}\right)$ \\
\hline 13 & $\left(A_{1} \cap B_{0}^{c},\left(A_{1}^{c} \cap B_{1}^{c}\right) \cup A_{0} \cup B_{0}\right)$ \\
\hline 14 & $\left(\left(A_{1} \cup B_{1}\right) \cap A_{0}^{c} \cap B_{0}^{c},\left(A_{1}^{c} \cap B_{1}^{c}\right) \cup A_{0} \cup B_{0}\right)$
\end{tabular}

Table 4. Conjunctions on orthopairs

can define a three valued set in an obvious way: $f(x)=1$ if $x \in A_{1} ; f(x)=0$ if $x \in A_{0}$ and $f(x)=\frac{1}{2}$ otherwise. So, we have a bijection between the collection of three-valued sets $\mathcal{F}_{\frac{1}{2}}(X):=\{f \mid f: X \mapsto \mathbf{3}\}$ and the collection of orthopairs of $X, \mathcal{O}(X):=\left\{\left(A_{1}, A_{0}\right) \mid A_{1}, A_{0} \in X ; A_{1} \cap A_{0}=\emptyset\right\}$.

We note that from $\left(A_{1}, A_{0}\right)$, another subset $A^{*}:=A_{0}^{c}$ of the universe can be defined as the negation of the falsity domain. In other words, renaming $A_{1}$ as $A_{*}$, an alternative representation of three valued sets is obtained by means of pairs of nested subsets $\left(A_{*}, A^{*}\right)$ of $X$, where $A_{*} \subseteq A^{*}$, which can be viewed as upper and lower approximations of some unknown set. We denote by $\mathcal{N}(X)$ the collection of nested pairs of subsets of $X$.

These constructions are known in the fuzzy set field: orthopairs can be viewed as special cases of so-called "intuitionistic fuzzy sets" 3 of Atanassov [3], and the nested version can be generalized to interval-valued fuzzy sets. They can be equipped with isomorphic structures [31].

Due to the bijection outlined above, we are able to translate all the operations from $\mathcal{F}(X)$ to $\mathcal{O}(X)$, and in particular all the 14 implications and conjunctions defined above. They are listed in Tables 4 and 6 . Note that the operations are often easier to understand using nested pairs as seen on Tables 5 and 7.

The three negations, respectively the involutive, intuitionistic and paraconsistent ones, take the following forms:

$$
\begin{aligned}
& \neg\left(A_{1}, A_{0}\right)=\left(A_{0}, A_{1}\right) ; \\
& \sim\left(A_{1}, A_{0}\right)=\left(A_{0}, A_{0}^{c}\right) ; \\
& -\left(A_{1}, A_{0}\right)=\left(A_{1}^{c}, A_{1}\right) .
\end{aligned}
$$

\footnotetext{
${ }^{3}$ where the word intuitionistic does not have the usual meaning [34].
} 


\begin{tabular}{c|c}
$\mathrm{n}$ & $\left(A_{*}, A^{*}\right) *\left(B_{*}, B^{*}\right)$ \\
\hline 1 & $\left(A^{*} \cap B^{*}, A^{*} \cap B^{*}\right)$ \\
\hline 2 & $\left(\left(A_{*} \cup B_{*}\right) \cap A^{*} \cap B^{*}, A^{*} \cap B^{*}\right)$ \\
\hline 3 & $\left(A_{*} \cap B^{*}, A^{*} \cap B^{*}\right)$ \\
\hline 4 & $\left(A^{*} \cap B_{*}, A^{*} \cap B^{*}\right)$ \\
\hline 5 & $\left(A_{*} \cap B_{*}, A^{*} \cap B^{*}\right)$ \\
\hline 6 & $\left(A_{*} \cap B_{*}, A^{*} \cap B_{*}\right)$ \\
\hline 7 & $\left(A_{*} \cap B_{*}, A_{*} \cap B_{*}\right)$ \\
\hline 8 & $\left(A_{*} \cap B_{*}, A_{*} \cap B^{*}\right)$ \\
\hline 9 & $\left(A^{*} \cap B_{*},\left(A_{*} \cup B_{*}\right) \cap A^{*} \cap B^{*}\right)$ \\
\hline 10 & $\left(A_{*} \cap B_{*},\left(A_{*} \cup B_{*}\right) \cap A^{*} \cap B^{*}\right)$ \\
\hline 11 & $\left(A_{*} \cap B^{*}, A_{*} \cap B^{*}\right)$ \\
\hline 12 & $\left(A_{*} \cap B^{*},\left(A_{*} \cup B_{*}\right) \cap A^{*} \cap B^{*}\right)$ \\
\hline 13 & \\
\hline 14 & $\left(\left(A_{*} \cup B_{*}\right) \cap A^{*} \cap B^{*},\left(A_{*} \cup B_{*}\right) \cap A^{*} \cap B^{*}\right)$
\end{tabular}

Table 5. Conjunctions on nested pairs

So, in the case of orthopairs, the definition of connectives is just a matter of translation from Tables 1 and 2. However, if these orthopairs are viewed as illknown sets some difficulties with truth-functionality occur. In the case of rough sets, we encounter even more difficulties, as we are going to explain.

\section{Three-valued connectives on ill-known sets}

Ill-known sets are sets whose boundaries are ill-known, namely it is not known whether some elements belong to them or not. The neutral region is then an uncertainty region. A typical situation where ill-known sets are obtained is as follows [37,28]. Consider $X$ as a set of objects and $f$ a feature (or attribute) mapping : $X \rightarrow V$ where $V$ is the domain of the corresponding attribute. So, $\forall x \in X, f(x)$ is the attribute value of object $x$.

Suppose we want to describe the set of objects that satisfy a property represented by a subset $C \subset V$ of values. For instance $X$ is a set of persons, $f$ is the height, and $C$ means taller than $1.70 \mathrm{~m}$. The set of persons that satisfy the criterion $C$ is defined by $f^{-1}(C) \subset X$.

Suppose for some reason $f(x)$ is not always known precisely. Let a one-tomany mapping $F: X \rightarrow \wp(V)$ represent an imprecise observation of the attribute $f$. Namely, for each object $x \in X$, all that is known about the attribute value $f(x)$ is that it belongs to the non-empty set $F(x) \subseteq V$. For instance, the heights of some persons $x$ are ill-known, and are described by the set $F(x)$ of (mutually exclusive) heights. Because of the incompleteness of the information, the subset $A=f^{-1}(C) \subseteq X$ of objects that satisfy the criterion $C$ is an "ill-known set" [37]. Let us first recall the following definition:

Definition 3.1. ([30]) Let $X$ and $V$ be two arbitrary sets and let $F: X \rightarrow \wp(V)$ be a multi-valued mapping with non-empty images. Let $C \subseteq V$ be an arbitrary 


\begin{tabular}{c|c|c}
$\mathrm{n}$ & $\left(A_{1}, A_{0}\right) \Rightarrow\left(B_{1}, B_{0}\right)$ & \\
\hline 1 & $\left(A_{0}^{c} \rightarrow B_{1},\left(A_{0}^{c} \rightarrow B_{1}\right)^{c}\right)$ & Sette \\
\hline 2 & $\left(A_{0}^{c} \rightarrow B_{1},\left[\left(A_{1} \rightarrow B_{1} \cap \cap\left(A_{0}^{c} \rightarrow B_{0}^{c}\right)\right]^{c}\right)\right.$ & Sobociński \\
\hline 3 & $\left(A_{0}^{c} \rightarrow B_{1},\left(A_{1} \rightarrow B_{1}\right)^{c}\right)$ & \\
\hline 4 & $\left(A_{0}^{c} \rightarrow B_{1},\left(A_{0}^{c} \rightarrow B_{0}^{c}\right)^{c}\right)$ & Jaśkowski \\
\hline 5 & $\left(A_{0}^{c} \rightarrow B_{1},\left(A_{1} \rightarrow B_{0}^{c}\right)^{c}\right)$ & Kleene \\
\hline 6 & $\left(A_{0}^{c} \rightarrow B_{0}^{c},\left(A_{0}^{c} \rightarrow B_{0}^{c}\right)^{c}\right)$ & \\
\hline 7 & $\left(A_{0}^{c} \rightarrow B_{0}^{c},\left(A_{1} \rightarrow B_{0}^{c}\right)^{c}\right)$ & Bochvar \\
\hline 8 & $\left(A_{1} \rightarrow B_{0}^{c},\left(A_{1} \rightarrow B_{0}^{c}\right)^{c}\right)$ & Nelson \\
\hline 9 & $\left(A_{1} \rightarrow B_{1},\left(A_{1} \rightarrow B_{0}^{c}\right)^{c}\right)$ & Gödel \\
\hline 10 & $\left(\left(A_{1} \rightarrow B_{1}\right) \cap\left(A_{0}^{c} \rightarrow B_{0}^{c}\right),\left(A_{0}^{c} \rightarrow B_{0}^{c}\right)^{c}\right)$ & Łukasiewicz \\
\hline 11 & $\left.\left(A_{1} \rightarrow B_{1}\right) \cap\left(A_{0}^{c} \rightarrow B_{0}^{c}\right),\left(A_{1} \rightarrow B_{0}^{c}\right)^{c}\right)$ & \\
\hline 12 & $\left(A_{1} \rightarrow B_{1},\left(A_{1} \rightarrow B_{1}\right)^{c}\right)$ & Gaines-Rescher
\end{tabular}

Table 6. Implications on orthopairs, where $A \rightarrow B=A^{c} \cup B$

subset of $Y$. The upper inverse of $C$ is defined as $F^{*}(C)=\{x \in X: F(x) \cap C \neq$ $\emptyset\}$. The lower inverse of $C$ is defined as $F_{*}(C)=\{x \in X: F(x) \subseteq C\}$.

According to this definition, $A=f^{-1}(C)$ can be approximated from above and from below, respectively, by upper and lower inverses of $C$ via $F$ :

- $A^{*}=F^{*}(C)$ is the set of objects that possibly belong to $A=f^{-1}(C)$.

$-A_{*}=F_{*}(C)$ is the set of objects that surely belong to $A=f^{-1}(C)$.

The interval $\left[A_{*}, A^{*}\right]=\left\{B, A_{*} \subseteq B \subseteq A^{*}\right\}$ in the Boolean algebra, called an interval set by Yao [73], contains the ill-known set $A$. Alternatively, we can consider orthopairs $\left(A_{1}, A_{0}\right)$ such that $\left[A_{*}, A^{*}\right]=\left\{B: A_{1} \subseteq B, A_{0} \cap B \neq \emptyset\right\}$.

If pairs of sets represent constraints on ill-known sets, we would like to compute the knowledge we may have on the result of combining two ill-known sets $A$ and $B$ by means of a three-valued connective merging their approximations $\left(A_{1}, A_{0}\right)$ and $\left(B_{1}, B_{0}\right)$. What is aimed at is, for any Boolean connective $c$, to find the orthopair $\left(c(A, B)_{1}, c(A, B)_{0}\right)$ representing our knowledge about $c(A, B)$ in the form $c_{3}\left(\left(A_{1}, A_{0}\right),\left(B_{1}, B_{0}\right)\right)$ where $c_{3}$ is a three-valued extension of $c$.

Consider $\frac{1}{2}$ as the set $\{0,1\}$ (understood as an interval such that $0<1$ ), the other "intervals" being the singletons $\{0\}$ and $\{1\}$. We can define connectives on ill-known sets by extending the Boolean connectives to such three-valued sets understood as interval-valued sets. Indeed this comes down to the following computations [33, 53]:

- For conjunction : $\{0\} \wedge\{0,1\}=\{0 \wedge 0,0 \wedge 1\}=\{0\}$;

$\{1\} \wedge\{0,1\}=\{1 \wedge 0,1 \wedge 1\}=\{0,1\}$, etc.

- For disjunction : $\{0\} \vee\{0,1\}=\{0 \vee 0,0 \vee 1\}=\{0,1\}$;

$\{1\} \vee\{0,1\}=\{1 \vee 0,1 \vee 1\}=\{1\}$, etc.

- For negation: $\neg\{0,1\}=\{\neg 0, \neg 1\}=\{0,1\}$. 


\begin{tabular}{c|c|c}
$\mathrm{n}$ & $\left(A_{*}, A^{*}\right) \Rightarrow\left(B_{*}, B^{*}\right)$ & \\
\hline 1 & $\left(A^{*} \rightarrow B_{*}, A^{*} \rightarrow B_{*}\right)$ & Sette \\
\hline 2 & $\left(A^{*} \rightarrow B_{*},\left(A_{*} \rightarrow B_{*}\right) \cap\left(A^{*} \rightarrow B^{*}\right)\right)$ & Sobociński \\
\hline 3 & $\left(A^{*} \rightarrow B_{*}, A_{*} \rightarrow B_{*}\right)$ & \\
\hline 4 & $\left(A^{*} \rightarrow B_{*}, A^{*} \rightarrow B^{*}\right)$ & Jaśkowski \\
\hline 5 & $\left(A^{*} \rightarrow B^{*}, A^{*} \rightarrow B^{*}\right)$ & Kleene \\
\hline 6 & $\left(A^{*} \rightarrow B^{*}, A_{*} \rightarrow B^{*}\right)$ & \\
\hline 7 & $\left(A_{*} \rightarrow B^{*}, A_{*} \rightarrow B^{*}\right)$ & Bochvar \\
\hline 8 & $\left(A_{*} \rightarrow B_{*}, A_{*} \rightarrow B^{*}\right)$ & Nelson \\
\hline 9 & $\left(\left(A_{*} \rightarrow B_{*}\right) \cap\left(A^{*} \rightarrow B^{*}\right), A^{*} \rightarrow B^{*}\right)$ & Gödel \\
\hline 10 & $\left.\left(A_{*} \rightarrow B_{*}\right) \cap\left(A^{*} \rightarrow B^{*}\right), A_{*} \rightarrow B^{*}\right)$ & \\
\hline 11 & $\left(\left(A_{*} \rightarrow B_{*}, A_{*} \rightarrow B_{*}\right) \cap\left(A^{*} \rightarrow B^{*}\right), A_{*} \rightarrow B_{*}\right)$ & Eukasiewicz \\
\hline 12 & & Gaines-Rescher
\end{tabular}

Table 7. Implications on nested pairs, where $A \rightarrow B=A^{c} \cup B$

The set 3 of non-empty intervals on $\{0,1\}$, equipped with the interval extension of classical connectives is isomorphic to a three-valued Kleene algebra.

However, using such connectives of Kleene logic to compute a combination of ill-known sets only captures an approximation of the actual result. For instance, even if $A$ is ill-known, $A \cap A^{c}=\emptyset((\emptyset, X)$ in terms of orthopairs). However, if $\left(A_{1}, A_{0}\right)$ are constraints on some unknown set $A$, the orthopair approximation of $A^{c}$ is $\left(A_{0}, A_{1}\right)$, but, applying the Kleene conjunction $\left(A_{1}, A_{0}\right) \cap_{5}\left(A_{0}, A_{1}\right)=$ $\left(A_{1} \cap A_{0}, A_{1} \cup A_{0}\right)$ is an imperfect approximation of the expected result $(\emptyset, X)$ since the former is equal to $\left(\emptyset, A_{1} \cup A_{0}\right)$.

So we should get $\left(A_{1}, A_{0}\right) \cap\left(A_{0}, A_{1}\right)=(\emptyset, X)$ using an appropriate conjunction. This result can be obtained using conjunctions $\cap_{i}, i>5$ by checking Table 4 (or Table 5 in terms of nested pairs). But then note that while one expects $\left(A_{1}, A_{0}\right) \cap\left(A_{1}, A_{0}\right)=\left(A_{1}, A_{0}\right)$ this is what is obtained on Table 4 (or Table 5 in terms of nested pairs) only for $\cap_{i}, i=2,3,4,5$. So none of the 14 reasonable conjunctions can provide the expected results.

In conclusion, the use of three-valued connectives to reason about ill-known sets looks hopeless: it is not the same to reason truth-functionally on objects made of pairs of sets, and to exploit pairs of sets viewed as constraints on a illknown set $A \in\left\{B: A_{1} \subseteq B, A_{0} \cap B \neq \emptyset\right\}$ : the former is a coarse approximation of the latter. Note that the same kind of critique applies to interval-valued fuzzy sets where it is often proposed interval extensions of basic connectives [33, 40] to handle uncertainty about gradual membership.

\section{Three-valued connectives on rough sets}

A rough set clearly defines a three-valued set, since it can be viewed as an upper and a lower approximation of a set. It was tempting to search for an 
algebra of rough sets from the three-valued logic literature. The problem of defining a three-valued logic (and especially an implication) for rough sets has been addressed by several authors. Łukasiewicz and Gödel implications have been introduced in rough sets by Pagliani [59] and for abstract versions of rough approximations in [18]. Pagliani also studied rough sets from the standpoint of Nelson algebras [58] and used Nelson implication. Eukasiewicz logic was also considered as the proper setting for rough sets by Banerjee [9] and Iturrioz [49]. On the other hand the Gaines-Rescher implication is the one adopted in [11] and Kleene implication in [22]. I. Düntsch in [41] introduced a propositional logic for rough set whose algebraic counterpart are double Stone algebras. The objects of this logic are nested pairs of the form $\left(A_{*}, A^{*}\right)$ and the implication considered is the Gödel one. For a general overview of algebraic structures related to rough sets we refer to [10].

A different and new approach is presented in [6], where the non-deterministic behaviour of rough sets is brought directly into a logical calculus. Indeed, the semantics of the implication is given by the non-deterministic NMatrix of Table 8 .

\begin{tabular}{c|c|c|c}
$\rightarrow$ & 0 & $\frac{1}{2}$ & 1 \\
\hline 0 & 1 & 1 & 1 \\
\hline$\frac{1}{2}$ & $\frac{1}{2}$ & $\left\{\frac{1}{2}, 1\right\}$ & 1 \\
\hline 1 & 0 & $\frac{1}{2}$ & 1
\end{tabular}

Table 8. Non-deterministic implication

Clearly, as the authors point out, the two "determinazations" of this situation correspond to Kleene and Eukasiewicz implication. Of course, the problem of non-determinism still remains, it is just shifted on a different level. And while this approach may look "less truth-functional" than the usual ones, its completeness with respect to the calculus of rough sets is unclear.

In this section we study the compatibility between the calculus of rough sets and three-valued connectives. We show that formally, it is possible to express three-valued logic connectives in terms of combinations of rough sets. But our results make it clear that the practical significance of these mathematical results is questionable.

\subsection{Some basics of rough sets}

In constrast with the scenario for ill-known sets, the starting point of rough sets is usually a set of data about some objects gathered in a so called Information Table (see for instance [61]).

Definition 4.1. An Information Table is a structure $\mathcal{K}(X)=\langle X, A$, val, $f\rangle$ where: 
- the universe $X$ is a non empty set of objects;

- A is a non empty set of attributes;

- val is the set of all possible values that can be observed for all attributes;

- $f$ (called the information map) is a mapping $X \times A \rightarrow$ val which associates to any pair object $x \in X$ and attribute $a \in A$, the value $f(x, a) \in$ val assumed by a for the object $x$.

On an Information Table, we define an Indiscernibility relation among objects as

$$
x R y \quad \text { iff } \quad \forall a \in A f(x, a)=f(y, a)
$$

The indiscernibility relation is an equivalence relation (reflexive, symmetric, transitive) that partitions the universe into equivalence classes:

$$
[x]_{R}=\{y: x R y\}
$$

In the following, we abstract from the notion of Information Table and suppose that an (equivalence) relation is available on a set of objects.

Definition 4.2. An approximation space is a pair $(X, R)$ with $X$ a set of objects and $R$ an equivalence relation on $X$.

On any approximation space, it is possible to define the lower and upper approximation of a given set.

Definition 4.3. Let $(X, R)$ be an approximation space. The lower approximation of $A \subseteq X$ is

$$
l_{R}(A):=\left\{x \in X \mid[x]_{R} \subseteq A\right\}
$$

and the upper approximation of $A$ is

$$
u_{R}(A):=\left\{x \in X \mid[x]_{R} \cap A \neq \emptyset\right\} \supseteq l_{R}(A)
$$

$A$ rough set is the lower-upper pair $r(A):=\left(l_{R}(A), u_{R}(A)\right)$ or equivalently the lower-exterior pair $r_{e}(A):=\left(l_{R}(A), e_{R}(A)\right):=\left(l_{R}(A), u_{R}^{c}(A)\right)$.

A set $A$ is said to be exact iff $l_{R}(A)=A$ or equivalently $A=u_{R}(A)$. We denote by $R S(X)$ the collection of all lower-upper approximations on $\mathrm{X}$ and by $R S_{e}(X)$ the set of lower-exterior approximations. The lower and upper approximations satisfy some interesting and useful properties. We list here some of them which will be useful later on.

Lemma 4.1. Let $(X, R)$ be an approximation space, and $A, B \subseteq X$. Then, the following properties hold.

1. $l_{R}(A \cap B)=l_{R}(A) \cap l_{R}(B)$;

2. $u_{R}(A \cup B)=u_{R}(A) \cup u_{R}(B)$;

3. If one of $A, B$ is exact then $l_{R}(A) \cup l_{R}(B)=l_{R}(A \cup B)$ and $u_{R}(A \cap B)=$ $u_{R}(A) \cap u_{R}(B)$

4. $l_{R}(A) \subseteq A \subseteq u_{R}(A)$; 
5. $l_{R}\left(l_{R}(A)\right)=l_{R}(A), u_{R}\left(u_{R}(A)\right)=u_{R}(A)$;

6. $l_{R}(A)=u_{R}^{c}\left(A^{c}\right)$.

Property 3 does not hold for two non-exact sets, that is $l_{R}(A) \cup l_{R}(B) \neq l_{R}(A \cup B)$ and $u_{R}(A \cap B) \neq u_{R}(A) \cap u_{R}(B)$ in this case. Otherwise stated, $l, u$ are not truthfunctional operators [74]; we can say we miss truth-functionality at the "internal level".

On the other hand, what we can try to do is to define truth-functional operators on rough sets viewed as upper-lower pairs $\left(A_{*}, A^{*}\right) \in R S(X)$, irrespective of the original underlying set. Then we say we have truth-functionality at the "external level". As we will see, this is feasible. Indeed, the lower-upper pair is clearly a nested pair and thus we can carry to this subcase the considerations on operations of the previous section. However, rough sets form a proper subset of nested pairs in the sense that every rough set induces a nested pair of sets in $X$, generated by a subset $H$ of $X$ through operators $l_{R}, u_{R}$, as $\left(l_{R}(H), u_{R}(H)\right) \in R S(X)$ but not vice versa [16]. Noticeably, no singleton $\{x\}$ can appear as an equivalence class in the boundary of a rough set, since either $x \in H$ and $\{x\}_{R} \subset l_{R}(H)$ or $x \notin H$ and $\{x\}_{R} \subset u_{R}(H)^{c}$.

So a truth-functional operation on orthopairs cannot be simply applied to rough sets. It must be shown that the operation is meaningful, that is:

- closed on the collection of all rough sets $R S(X)$ (or equivalently $R S_{e}(X)$ )

- related to a well-defined combination of the underlying (Boolean) approximated sets.

As we will see, in this process, some interpretability problems of the connectives arise.

\subsection{Rough sets and external truth-functionality}

Since $R S(X) \subset \mathcal{N}(X)$, the first question is whether, once we restrict to $R S(X)$, the implications definable on $\mathcal{N}(X)$ are closed on $R S(X)$. In other words:

If $\odot$ is a three-valued binary operation on pairs $\left(A_{*}, A^{*}\right),\left(B_{*}, B^{*}\right) \in R S(X)$, and $A_{*}=l_{R}(A), A^{*}=u_{R}(A), B_{*}=l_{R}(B), B^{*}=u_{R}(B)$ for some $A, B \subset X$, does there exist an operation $\cdot$ on $2^{X}$ such that

$$
\left(l_{R}(A \cdot B), u_{R}(A \cdot B)\right)=\left(A_{*}, A^{*}\right) \odot\left(B_{*}, B^{*}\right) ?
$$

The answer is not straightforward, since first of all not all nested pairs $(A, B)$ can be generated by a subset $H$ of the universe as $\left(l_{R}(H), u_{R}(H)\right)$, as pointed out before. Moreover it must be clear that the relation $R$, used to build the partition and then to compute the approximation, is fixed in the above statement.

Let us start from already known results for basic operations [22]. First of all the negation of a set. This case is simple, indeed we have in terms of orthopairs:

$$
r\left(A^{c}\right)=\left(l_{R}\left(A^{c}\right), u_{R}\left(A^{c}\right)\right)=\left(u_{R}^{c}(A), l_{R}^{c}(A)\right)=r^{c}(A)
$$

Thus, the approximation of $A^{c}$ can be obtained by the approximation of $A$ in a truth-functional way. 
In case of intersection $r(A \cap B)$ and union $r(A \cup B)$, corresponding to the min conjunction 5 in Table 4 and the dual disjunction, consider Kleene conjunction and disjunction. Namely, we ask if there exist two sets $C, D \subseteq X$ such that

$$
\begin{aligned}
& r(C)=r(A) \sqcap r(B):=\left(l_{R}(A) \cap l_{R}(B), u_{R}(A) \cap u_{R}(B)\right) \\
& r(D)=r(A) \sqcup r(B):=\left(l_{R}(A) \cup l_{R}(B), u_{R}(A) \cup u_{R}(B)\right)
\end{aligned}
$$

At least three solutions were proposed in the literature. Bonikowski in [16] showed that the set $C$ can be built according to the following procedure:

1. If $u_{R}(A) \cap u_{R}(B)=\emptyset$ then $C=\emptyset$, else $u_{R}(A) \cap u_{R}(B)$ is of the form $\left[x_{1}\right] \cup \ldots \cup\left[x_{k}\right]$, where $\left[x_{i}\right]$ are equivalence classes of $R$.

2. Choose $y_{i} \in\left[x_{i}\right]$ for all $i$ such that $\left[x_{i}\right] \nsubseteq l_{R}(A) \cap l_{R}(B)$ (in the boundary) and build $Y=\left\{y_{i}: y_{i} \in\left[x_{i}\right] \nsubseteq l_{R}(A) \cap l_{R}(B)\right\}$

3. Finally, $C=\left[l_{R}(A) \cap l_{R}(B)\right] \cup Y$ (disjoint union).

Note that $l_{R}(C)=l_{R}(A \cap B)$, since no equivalence class $\left[x_{i}\right]$ in the boundary can be a singleton $\left\{y_{i}\right\}$, any $y_{i} \in Y$ is an element of a larger equivalence class, and so, $l_{R}(Y)=\emptyset$. The set $D$ for disjunction in (3) is computed with the same procedure applied to $A^{c}$ and $B^{c}$.

In [45] we can find another definition of internal intersection and union

$$
\begin{aligned}
& A \cap_{1} B=A \cap\left[l_{R}(B) \cup\left(B \cap u_{R}(A)^{c}\right) \cup\left(u_{R}(B) \cap l_{R}(A)^{c} \cap A\right) \cup\left(l_{R}(A) \cap B\right)\right] \\
& A \cup_{1} B=A \cup\left[l_{R}(B) \cup\left(B \cap u_{R}(A)^{c}\right) \cup\left(u_{R}(B) \cap l_{R}(A)^{c} \cap A\right) \cup\left(l_{R}(A) \cap B\right)\right]
\end{aligned}
$$

and again, $r\left(A \cap_{1} B\right)=r(A) \sqcap r(B), r\left(A \cup_{1} B\right)=r(A) \sqcup r(B)$. Finally, in [11], the following alternative solution has been proposed.

$$
\begin{aligned}
& A \cap_{2} B=(A \cap B) \cup\left(\left(A \cap u_{R}(B)\right) \cap\left(u_{R}(A \cap B)^{c}\right)\right) \\
& A \cup_{2} B=(A \cup B) \cap\left(\left(A \cup l_{R}(B)\right) \cup\left(l_{R}(A \cup B)^{c}\right)\right)
\end{aligned}
$$

Note that $A \cap_{1} B$ and $A \cap_{2} B$ can be written as $\left[l_{R}(A) \cap l_{R}(B)\right] \cup Y^{\prime}$, where $Y^{\prime}$ is the union of proper subsets $Y_{i}$ of equivalence classes $\left[x_{i}\right]$ not in the intersection of the lower images. So, they are very close to one of the possible solutions of Bonikowski's procedure. Moreover, any solution has this form.

Proposition 4.1. Any set $C$ whose upper and lower approximations are respectively the intersections of the upper and of the lower approximation of $A$ and $B$ is of the form $\left[l_{R}(A) \cap l_{R}(B)\right] U Y^{\prime}$

Proof. Indeed, if $\mathrm{Y}^{\prime}$ does not contain at least one element of an equivalence class outside the intersection of the lower approximations and inside the intersection of their upper approximation, then the upper approximation of $\mathrm{C}$ is not the intersection of the upper approximations of $\mathrm{A}$ and $\mathrm{B}$. If $\mathrm{Y}^{\prime}$ contains one equivalence class outside the intersection of lower approximations and inside their union, then its lower approximation is larger than the intersection of lower approximations of $\mathrm{A}$ and $\mathrm{B}$. 
Now, we want to extend these definability results to all other three-valued operations introduced in the previous Section 2. Let us start from the negations whose proof is straightforward.

\section{Proposition 4.2 .}

$$
\begin{aligned}
& \neg\left(l_{R}(A), u_{R}(A)\right)=\left(u_{R}^{c}(A), l_{R}^{c}(A)\right)=r\left(A^{c}\right) ; \\
& \sim\left(l_{R}(A), u_{R}(A)\right)=\left(u_{R}^{c}(A), u_{R}^{c}(A)\right)=r\left(l_{R}\left(A^{c}\right)\right) ; \\
& -\left(l_{R}(A), u_{R}(A)\right)=\left(u_{R}\left(A^{c}\right), u_{R}\left(A^{c}\right)\right)=r\left(u_{R}\left(A^{c}\right)\right) .
\end{aligned}
$$

Now, as far as implications are concerned, some of them have already been studied in literature and it has been shown that they are closed on rough sets. These results are summarized in the following proposition.

\section{Proposition 4.3.}

$$
\begin{aligned}
& r(A) \Rightarrow_{5} \quad r(B)=r\left((A \rightarrow B) \cap\left(\left(A \rightarrow l_{R}(B)\right) \cup\left(l_{R}(A \rightarrow B)^{c}\right)\right)\right) ; \\
& r(A) \Rightarrow_{9} r(B)=r\left(l_{R}(A) \rightarrow B\right) ; \\
& r(A) \Rightarrow_{10} r(B)=r\left(\left(u_{R}(A) \rightarrow B\right) \cup\left[l_{R}(A) \rightarrow u_{R}^{c}(B)\right]^{c}\right) ; \\
& r(A) \Rightarrow_{11} r(B)=r\left(\left(l_{R}(A) \rightarrow B\right) \cap\left(A \rightarrow u_{R}(B)\right)\right) ; \\
& r(A) \Rightarrow_{14} r(B)=r\left(\left(\left(u_{R}(A) \rightarrow u_{R}(B)\right) \cap\left(l_{R}(A) \rightarrow l_{R}(B)\right)\right) .\right.
\end{aligned}
$$

Proof. The cases 5,10,11, respectively Kleene, Gödel and Łukasiewicz implications, are proved in [22]. The Nelson (case 9) implication immediately follows by its definition (and see also [59]). Finally, the Gaines-Rescher implication 14 has been studied in [11], where it is defined as

$$
r(A) \Rightarrow r(B)=(\neg \square r(A) \cup \square r(B)) \cap(\neg \diamond r(A) \cup \diamond r(B)),
$$

with $\square r(H)=\left(l_{R}(H), l_{R}(H)\right)$ and $\diamond r(H)=\left(u_{R}(H), u_{R}(H)\right)$. So, first of all, let us note that equation 4 is equivalent to the one in Table 7 , as can be easily proven. Then, from the definition in [11], we have $r(A) \Rightarrow_{14} r(B)=\left[r\left(l_{R}^{c}(A)\right) \sqcup\right.$ $\left.r\left(l_{R}(B)\right)\right] \sqcap\left[r\left(u_{R}^{c}(A)\right) \sqcup r\left(u_{R}(B)\right)\right]$ from which we arrive at the thesis.

In order to study the other implications and conjunctions, the following result concerning the application of the transformations (1) can be given.

Proposition 4.4. Let $\odot$ be a closed operation on $\mathcal{R}(X)$. Then, also a $[\mathcal{A}(\odot)]$, $a[\mathcal{V}(\odot)] b, a[\mathcal{S}(\odot)] b$ are closed on $\mathcal{R}(X)$.

Proof. The case of $\mathcal{A}(\odot)$ is trivial since it is the same operation as $\odot$ with different arguments. Operations $\mathcal{V}(\odot)$ and $\mathcal{S}(\odot)$ are a composition of $\odot$ and involutive negation $\neg$ which is closed by proposition 4.2 . So, we will have that $r(X)[\mathcal{V}(\odot)] r(Y)=r\left(Y^{c}\right) \odot r\left(X^{c}\right)$ and $r(X)[\mathcal{S}(\odot)] r(Y)=\neg\left[r(X) \odot r\left(Y^{c}\right)\right]$.

By the above propositions, we immediately get that also other implications and conjunctions are well defined, since as shown in $[23,25]$ they can be obtained by equation system (1) from the above implications in Proposition 4.3. 
Corollary 4.1. Conjunctions $7,9,10,11,13,14$ are closed on $\mathcal{R}(X)$ and the following hold:

$$
\begin{aligned}
& r(A) *_{7} \quad r(B)=r\left(A \cap l_{R}(B)\right) ; \\
& r(A) *_{9} \quad r(B)=r\left(l_{R}(A) \cap B\right) ; \\
& r(A) *_{10} r(B)=r\left(\left[l_{R}(A) \cup l_{R}(B)\right] \cap u_{R}(A) \cap B\right) ; \\
& r(A) *_{11} r(B)=r\left(\left[l_{R}(A) \cup l_{R}(B)\right] \cap A \cap B\right) ; \\
& r(A) *_{13} r(B)=r\left(\left[l_{R}(A) \cup l_{R}(B)\right] \cap A \cap u_{R}(B)\right) ; \\
& r(A) *_{14} r(B)=r\left(\left(l_{R}(A) \cup l_{R}(B)\right) \cap u_{R}(A) \cap u_{R}(B)\right) .
\end{aligned}
$$

Further, implications 7,13 are closed on $\mathcal{R}(X)$ and we have:

$$
\begin{aligned}
& r(A) \Rightarrow_{7} \quad r(B)=r\left(A \rightarrow u_{R}(B)\right) ; \\
& r(A) \Rightarrow_{13} r(B)=r\left(A \rightarrow l_{R}(B) \cup\left[l_{R}^{c}(A) \rightarrow u_{R}^{c}(B)\right]^{c}\right) .
\end{aligned}
$$

We now prove that all the remaining implications and conjunctions are closed on $\mathcal{R S}(X)$.

\section{Proposition 4.5.}

$$
\begin{aligned}
& r(A) \Rightarrow_{1} r(B)=r\left(u_{R}(A) \rightarrow l_{R}(B)\right) ; \\
& r(A) \Rightarrow_{2} r(B)=r\left(\left[A \rightarrow l_{R}(B)\right] \cap\left[u_{R}(A) \rightarrow B\right]\right) ; \\
& r(A) \Rightarrow_{3} r(B)=r\left(A \rightarrow l_{R}(B)\right) ; \\
& r(A) \Rightarrow_{6} r(B)=r\left(u_{R}(A) \rightarrow u_{R}(B)\right) ; \\
& r(A) \Rightarrow_{8} r(B)=r\left(l_{R}(A) \rightarrow u_{R}(B)\right) .
\end{aligned}
$$

Proof. Only $\Rightarrow_{2}$ deserves some explanation, the others being trivial. By Table 6 , we get $\left(l_{R}(A), u_{R}(A)\right) \Rightarrow_{2}\left(l_{R}(B), u_{R}(B)\right)=\left(u_{R}^{c}(A) \cup l_{R}(B),\left(u_{R}(B) \cup\right.\right.$ $\left.\left.u_{R}^{c}(A)\right) \cap\left(l_{R}^{c}(A) \cup l_{R}(B)\right)\right)$, which can be re-written as $\left(\left(u_{R}^{c}(A) \cup l_{R}(B)\right) \cap\left(u_{R}^{c}(A) \cup\right.\right.$ $\left.\left.l_{R}(B)\right),\left(u_{R}(B) \cup u_{R}^{c}(A)\right) \cap\left(l_{R}^{c}(A) \cup l_{R}(B)\right)\right)$. Applying equations (3), we obtain $\left[r\left(A^{c}\right) \sqcup r\left(l_{R}(B)\right)\right] \sqcap\left[r\left(l_{R}\left(A^{c}\right)\right) \sqcup r(B)\right]$ and by Lemma 4.1 we have the thesis.

Based on the implications in Proposition 4.5, it is possible to construct other conjunctions and implications (see $[23,25]$ ). So, due to Proposition 4.4 the following corollary holds.

Corollary 4.2. Conjunctions $1,2,3,4,6,8,12$ are closed on $\mathcal{R}(X)$ and the following hold:

$$
\begin{array}{ll}
r(A) *_{1} & r(B)=r\left(u_{R}(A) \cap u_{R}(B)\right) ; \\
r(A) *_{2} & r(B)=r\left(\left[A \cap u_{R}(B)\right] \cup\left[u_{R}(A) \cap B\right]\right) ; \\
r(A) *_{3} & r(B)=r\left(A \cap u_{R}(B)\right) \\
r(A) *_{4} & r(B)=r\left(u_{R}(A) \cap B\right) \\
r(A) *_{6} & r(B)=r\left(u_{R}(A) \cap l_{R}(B)\right) ; \\
r(A) *_{8} & r(B)=r\left(l_{R}(A) \cap l_{R}(B)\right) ; \\
r(A) *_{12} & r(B)=r\left(l_{R}(A) \cap u_{R}(B)\right) .
\end{array}
$$


Implications 4, 12 are closed on $\mathcal{R}(X)$ and the following hold:

$$
\begin{aligned}
& r(A) \Rightarrow_{4} r(B)=r\left(u_{R}(A) \rightarrow B\right) ; \\
& r(A) \Rightarrow_{12} r(B)=r\left(l_{R}(A) \rightarrow l_{R}(B)\right) .
\end{aligned}
$$

The above results show that the original question of finding subsets of $X$ that underlie all 14 three-valued conjunctions and implications applied to upper and lower approximations of sets in the sense of rough sets can be answered in the affirmative. However the reader may observe that the definition of such subsets, the approximations of which are constructed by such connectives, always involve lower and/or upper approximations of the two underlying sets to be combined.

\subsection{The interpretability of external truth-functional operations on rough sets}

In [22], we started an investigation on the significance of existing truth-functional three valued logics of ill-known sets described by pairs of disjoint (or pairs of nested) subsets. This work strongly suggested that while, from a mathematical standpoint, such three-valued logics are consistent with a rough set view, their interpretation with respect to reasoning about the original data tables is questionable. The operators analyzed in that work were Kleene conjunction and disjunction $(\min / \max )$ on three values and three different implications: Eukasiewicz, Gödel and Kleene. However, the concerns already raised for these known connectives seem to carry over to all the 28 three-valued connectives recalled in this paper, as the results obtained here in the previous section indicate.

Let us consider two sets of items $A, B$ defined in extension, the approximations $r(A)$ and $r(B)$ of which we want to aggregate with one of the three-valued connectives laid bare in this paper, say $\odot$. Concerning the existence of a set $C$ such that $r(C)=r(A) \odot r(B)$, we have seen that such an underlying set $C$ always exists. However, $C$ does not depend exclusively on $A$ and $B$ but strongly depends on the partition chosen (that is on the equivalence relation $R$ of the approximation space and finally on the set of attributes of an Information Table) because it depends on the lower and/or upper approximations of $A$ and $B$ as well. Moreover, even inside the same partition, several choices of $C$ are possible.

This difficulty is due, in some sense, to the presence of two languages: the fine-grained one needed to distinguish elements of $X$ and the (more restricted) one based on the attributes of the information table, that only allows to describe approximations of any subset of such elements. Combining approximations of illknown sets $A$ and $B$ truth-functionally yields well-behaved pairs of nested sets, but the corresponding internal combination of $A$ and $B$ that makes the external truth-functional combination meaningful is problematic.

Indeed, in the setting of rough sets, $A$ and $B$ are known in extension (they are in some sense the actual entities referred to) whereas, using the coarser attribute language instrumental to describe them, their intensions are available only through their approximations. So, the intension depends on the coarse language: the more (less) numerous the attributes, the finer (coarser) the description. Results in the previous section show that the set $C=A \cdot B$ displayed in 
the previous section for the 14 conjunctions and the 14 implications such that $r(A) \odot r(B)=r(A \cdot B)$ laid bare in this paper always depends on the partition induced by $R$ and so on the attributes defining the coarser language. Changing the coarser language (i.e., attributes in the Information Table) will alter the set $A \cdot B$ but not $A, B$. So, while we can interpret the external truth-functionality of operations on approximation pairs as providing approximation pairs of definable combinations of subsets of $X$, these subsets are definable only if the coarser language is fixed (in fact they need both languages, since $A \cdot B$ is potentially a Boolean set-theoretic combination of $\left.A, B, l_{R}(A), l_{R}(B), u_{R}(A), u_{R}(B)\right)$. As a consequence, we lose the interpretability of the results since these inner combinations are not intrinsic to $A$ and $B$, and depend on the indistinguishability relation.

\section{Rough Sets: From Modal Logic to Three-valued logics}

Apart from many-valued logics, a natural logical rendering of rough sets is through modal logics. This possibility has been addressed by several authors taking into account different variants of rough sets $[57,42,69,70,11,75,76,8$, 51]. This section provides some hints toward relating the three-valued and the modal logic views of rough sets, in connection with recent works translating three-valued logics into fragments of the modal logic KD.

\subsection{The standard modal approach to rough sets}

We now recall a modal logic for handling approximations of sets generated by an equivalence relation [56].

Its language $\mathcal{L}_{M}$ is the usual one of propositional logic plus necessity $\square$ and possibility $\diamond$. That is, we have a set of propositional variables $\mathcal{V}=\{a, b, c, \ldots\}$ and the connectives $\wedge,,^{\prime}, \square$. As usual, disjunction $\alpha \vee \beta$ stands for $\left(\alpha^{\prime} \wedge \beta^{\prime}\right)^{\prime}$, implication $\alpha \rightarrow \beta$ stands for $\alpha^{\prime} \vee \beta$, tautology $\top$ for $\alpha \vee \alpha^{\prime}$ and $\diamond \alpha=\left(\square \alpha^{\prime}\right)^{\prime}$. Well formed formulae are built in the standard way.

The axioms are those of propositional logic plus the axioms to characterize the modal connectives.

1. $\phi \rightarrow(\psi \rightarrow \phi)$

2. $(\psi \rightarrow(\phi \rightarrow \mu)) \rightarrow((\psi \rightarrow \phi) \rightarrow(\psi \rightarrow \mu))$

3. $\left(\phi^{\prime} \rightarrow \psi^{\prime}\right) \rightarrow(\psi \rightarrow \phi)$

(K) $\square(\alpha \rightarrow \beta) \rightarrow(\square \alpha \rightarrow \square \beta)$

(T) $\square \alpha \rightarrow \alpha$

(5) $\diamond \alpha \rightarrow \square \diamond \alpha$

Finally, rules are modus ponens and necessitation: If $\vdash \alpha$ then $\vdash \square \alpha$. The above system is called S5, and its semantics is in terms of equivalence relations [20]. It is thus the natural logical setting for rough sets [56].

The semantics is given through a model $M=(X, \mathcal{R}, v)$, where $(X, R)$ is an approximation space and $v$ is a mapping from formulae to $2^{X}$. In standard modal 
logic terminology, $X$ is the set of possible worlds, $R$ the accessibility relation and $v(\alpha)$ represents the set of possible worlds where $\alpha$ holds. The interpretation $v$ is recursively defined on propositional connectives as usual as:

$$
\begin{aligned}
v\left(\alpha^{\prime}\right) & =v(\alpha)^{c} \\
v\left(\alpha_{1} \wedge \alpha_{2}\right) & =v\left(\alpha_{1}\right) \cap v\left(\alpha_{2}\right) \\
v\left(\alpha_{1} \vee \alpha_{2}\right) & =v\left(\alpha_{1}\right) \cup v\left(\alpha_{2}\right)
\end{aligned}
$$

and modal operators are mapped to lower and upper approximations:

$v(\square \alpha)=L_{R}(v(\alpha))=\left\{x \in X:[x]_{R} \subseteq v(\alpha)\right\}=\{x \in X: \forall w, x \mathcal{R} w, w \in v(\alpha)\}$

$v(\diamond \alpha)=U_{R}(v(\alpha))=\left\{x \in X:[x]_{R} \cap v(\alpha) \neq \emptyset\right\}=\{x \in X: \exists w, x \mathcal{R} w, w \in v(\alpha)\}$

Note that, in the S5 approach, one can represent sets ("objective" formulae $\alpha$ ) and their lower $(\square \alpha)$ and upper $(\diamond \alpha)$ approximations.

This approach can easily be extended to rough set models based on a relation that is not necessarily an equivalence one $[75,76]$. Indeed, it is well known in modal logic [20] that, once fixed the basic axioms 1-3 and (K), then to any additional modal axiom according to Table 9 corresponds a specific property of the accessibility relation.

\begin{tabular}{c|c|c} 
Name & Axiom & Property \\
\hline $\mathrm{T}$ & $\square \alpha \rightarrow \alpha$ & Reflexive \\
4 & $\square \alpha \rightarrow \square \square(\alpha)$ & Transitive \\
5 & $\nabla \alpha \rightarrow \square(\diamond(\alpha))$ & Euclidean \\
$\mathrm{D}$ & $\square \alpha \rightarrow \diamond \alpha$ & Serial \\
$\mathrm{B}$ & $\alpha \rightarrow \square \diamond \alpha$ & Symmetric
\end{tabular}

Table 9. Correspondence between modal axioms and relation properties

Another extension of the basic approach is the logic DAL [42], meant to deal with approximation spaces with more than one equivalence relation $\left(X, R_{i}\right)$. Each relation represents a different attribute, for instance "having the same number of circles", "having the same number of crosses".

\subsection{The three-valued modal approach}

A different approach is given by the so-called Pre-Rough Logic (PRL) and its corresponding algebra called pre-rough algebra [11], which is based on a 3-valued logic. Atoms of the logic are three-valued entities, which represent nested approximation pairs. They can be obtained from the S5 logic of the previous section by considering a weaker notion of logical equivalence in S5. Namely, Banerjee and Chakraborty speak of rough equivalence of two propositional formulae $\alpha$ and $\beta$ whenever $\square \alpha$ is semantically equivalent to $\square \beta$ and $\diamond \alpha$ is semantically equivalent to $\diamond \beta$. They consider the result of quotienting the language with the rough 
equivalence relation, and each equivalence class corresponds to an approximation pair, which becomes a formula of the pre-rough logic PRL. Note that by doing so, the underlying set, the approximations of which are given by modal formulae, is lost: we can no longer distinguish between propositional formulae that are roughly equivalent.

For the sake of clarity, we denote by $\mu, \nu, \rho$ the formulae of PRL. Primitive connectives of the logic are negation, intersection and necessity, respectively denoted by $\neg, \wedge, \mathbf{\square}$, from which we derive the disjunction $\vee$ through de Morgan properties, the dual modality $\boldsymbol{\nabla} \mu=\neg \mathbf{\square} \mu$ and the implication as $\mu \rightarrow \nu=$ $(\neg \boldsymbol{\square} \mu \vee \boldsymbol{\square} \nu) \wedge(\neg \boldsymbol{\nabla} \mu \vee \vee \nu)$. The axioms of the logic are

RL1 $\mu \rightarrow \mu$

RL2 $\neg \neg \mu \leftrightarrow \mu$

RL3 $\mu \wedge \nu \rightarrow \mu$

$\mathrm{RL} 4 \mu \wedge \nu \rightarrow \nu \wedge \mu$

RL5 $\mu \wedge(\nu \vee \rho) \leftrightarrow(\mu \wedge \nu) \vee(\mu \wedge \rho)$

RL6 $\square \mu \rightarrow \mu$

$\operatorname{RL7} \boldsymbol{\square}(\mu \wedge \nu) \leftrightarrow \boldsymbol{\square} \mu \wedge \boldsymbol{\square} \nu$

RL8 $\square \mu \rightarrow \square \square \mu$

RL9 $\boldsymbol{\nabla} \mu \rightarrow \mathbf{\square} \mu$

$\mathrm{RL} 10 \mathbf{\square}(\mu \vee \nu) \leftrightarrow \mathbf{\square} \mu \vee \mathbf{\square} \nu$

A sequent calculus for this logic is provided by Sen and Chakraborty [64].

The semantics is three-valued, and some connectives are based on Kleene logic, using ternary valuations $t$ such that [11]

$$
\begin{aligned}
t(\neg \mu) & =\neg t(\mu) \text { (Kleene negation) } \\
t(\mu \wedge \nu) & =t(\mu) \sqcap t(\nu) \text { (Kleene conjunction) } \\
t(\boldsymbol{\square} \mu) & =\neg-t(\mu) \text { (using the paraconsistent negation }- \text { ) } \\
t(\mu \rightarrow \nu) & =(t(\neg \square \mu) \cup t(\mathbf{\square} \nu)) \cap(t(\neg \diamond \mu) \cup t(\diamond \nu))
\end{aligned}
$$

In connection with the S5-based rough set logic of the previous section, a nonmodal formula $\mu$ in PRL corresponds to an approximation pair $\left(A(\mu)_{*}, A(\mu)^{*}\right)$ over possible worlds in $X$ (Boolean interpretations), both $A(\mu)_{*}$ and $A(\mu)^{*}$ being exact sets of such valuations (formulae $\alpha$ such that $v(\square \alpha)=v(\diamond \alpha)$, whenever $v(\alpha)=A(\mu)_{*}$ or $\left.A(\mu)^{*}\right)$. The operator $\mathbf{\square}$ corresponds to extracting the core $A(\mu)_{*}$ of the three-valued set over $X$ induced by $\mu$. In terms of fuzzy sets, $\mathbf{\square} \mu$ correspond to the core of $\mu$ and $\diamond \mu$ correspond to its support. It can thus be easily seen that

$-t(\boldsymbol{\square} \mu)$ is two-valued and $\boldsymbol{\square} \mu$ corresponds to the (exact) approximation pair $\left(A(\mu)_{*}, A(\mu)_{*}\right)$;

- $t(\boldsymbol{\nabla} \mu)$ is two-valued and $\boldsymbol{\square} \mu$ corresponds to the (exact) approximation pair $\left(A(\mu)^{*}, A(\mu)^{*}\right)$

$-t(\mu \rightarrow \nu)$ is two-valued : it is Gaines-Rescher implication expressing the double inclusion of upper and lower approximations $A(\mu)_{*} \subseteq B(\nu)_{*}$ and $A(\mu)^{*} \subseteq B(\nu)^{*}$. 
In view of the above semantical considerations, RL6-RL10 are expected. RL6 and RL9 correspond to S5 axioms T, and 5 respectively, while RL7 and RL8 are valid in S5. However, due to RL10, and are deviant modalities, that are not trivial because the logic is 3 -valued [19].

The connection between S5 and PRL is maintained by noticing that an approximation pair $\left(A(\mu)_{*}, A(\mu)^{*}\right)$ underlies a crisp set $A$ of $X$ that is approximated by this pair. In order to maintain this view throughout all the formulae in PRL, Banerjee and Chakraborty make it clear what is the set approximated by for instance $\left(C(\mu \wedge \nu)_{*}, C(\mu \wedge \nu)^{*}\right)$ when $A$ and $B$ are the sets approximated by $\left(A(\mu)_{*}, A(\mu)^{*}\right)$ and $\left(B(\nu)_{*}, B(\nu)^{*}\right)$. They do it by introducing the intersection $A \cap_{2} B$ already discussed in Subsection 4.2, and that does not depend solely on $A$ and $B$.

The fact that in the PRL syntax, we no longer explicitly refer to the approximated set and maintain truth-functionality for evaluating formulae expressing approximation pairs is thus paid by the fact that it is no longer possible to intrinsically define the approximated set referred to by a compound PRL formula in terms of the approximated sets of its elementary sub-formulae. On the other hand, while the S5 setting avoids this pitfall, one may find it unrealistic to represent at the same time the approximated pairs with the approximated set in the language, as the point made by rough set theory is that sets are only described in intension through the available attributes, while their precise extension is out of reach. In this sense, while S5 seems to precisely capture the formal setting of rough sets, the PRL logic looks more faithful to the way rough sets can be used in practice, that is, it refers to the situation where we know the approximations, but neither the underlying set nor the equivalence relation. Unfortunately, the PRL rendering of rough set theory, and logical combinations of upper and lower approximations, in terms of a three-valued logic looks like an approximation as well.

Interestingly, connections between PRL and major three-valued logics have been laid bare:

- In [11] it is shown that the algebra of PRL (a bounded lattice structure $(A, 0,1)$ equipped with Kleene connectives $(\sqcup, \neg, \sqcap)$ the modality $\mathbf{\square}$ and Gaines-Rescher implication $\rightarrow_{14}$, is equivalent to semi-simple Nelson algebras, that is, a bounded lattice structure $(A, 0,1)$ equipped with Kleene connectives, the paraconsistent negation - and Nelson implication $\rightarrow_{9}$. Indeed they notice that $-\mu=\neg \boldsymbol{\square} \mu$ and $\mu \rightarrow_{9} \nu=\neg \boldsymbol{\square} \mu \sqcup \nu$. Conversely, $\boldsymbol{\square}=\neg-\mu$ and $\mu \rightarrow_{14} \nu=\neg-\left(\mu \rightarrow_{9} \nu\right)$.

- It has been proved in [9], that PRL is equivalent to three-valued Łukasiewicz logic. Especially, Banerjee points out that the Eukasiewicz implication can be written as $(\neg \mu \sqcup \nu) \sqcap(\neg \mu \sqcup \nu)$. Conversely, PRL connectives $\wedge, \vee, \diamond$ are defined as usual $\mu \sqcup \nu=\left(\left(\mu \rightarrow_{11} \nu\right) \rightarrow_{11} \nu\right), \mu \sqcap \nu=\neg(\neg \mu \sqcup \neg \nu)$ and $\diamond \mu=\neg\left(\mu \rightarrow_{11} \neg \mu\right)$.

But these findings are not surprising at all given the results in [25] whereby from Kleene connectives plus paraconsistent negation, one can reconstruct all 
28 monotonic three-valued conjunctions and implications that extend Boolean ones (as per the last item of Proposition 2.2).

\subsection{From three-valued rough set logic to modal logic}

We have seen two different modal approaches concerning rough sets: a Boolean one based on S5 and a many-valued one, the PRL logic, the latter being based on a clustering of roughly equivalent formulae of the former. Conversely, Banerjee [9] proves that PRL is embeddable in S5 in the sense that PRL formulae can be expressed as S5 formulae via a translation operation $(\cdot)^{\tau}$ such that $\vdash_{P R L} \mu$ iff $\vdash_{S 5} \mu^{\tau}$.

Namely, the PRL negation $\neg$ becomes the classical negation ', the PRL necessity operator $\square$ becomes the classical one $\square$, and

$$
(\mu \sqcap \nu)^{\tau}=\left(\mu^{\tau} \wedge \nu^{\tau}\right) \vee\left(\mu^{\tau} \wedge \diamond \nu^{\tau} \wedge\left(\diamond\left(\mu^{\tau} \wedge \nu^{\tau}\right)\right) .\right.
$$

The latter encodes the set supposedly upper and lower approximated by the intersection of upper and lower approximations of two sets, already met in previous sections as $\cap_{2}$. This translation can only yield a fragment of S5.

There is another way of capturing the semantics of three-valued logics in a modal setting, whenever the third truth-value stands for unknown $[24,26]$. It is enough to use a fragment of KD (or of S5) called MEL [12]. In particular, we can translate with three-valued Łukasiewicz logic L3 into MEL, while preserving L3 theorems. Since PRL is equivalent to L3, it is interesting to translate PRL into MEL as well.

The language MEL $[12,13]$ is a very limited fragment of the modal logic S5. It uses a sublanguage $\mathcal{L}_{\square}$ of S5 defined by encapsulating propositional formulae from a modality-free propositional language $\mathcal{L}$ (using the same notations as in Subsection 5.1):

$$
\mathcal{L}_{\square}=\square \alpha: \alpha \in \mathcal{L}|\neg \phi| \phi \wedge \psi|\phi \vee \psi| \phi \rightarrow \psi .
$$

Note that $\mathcal{L}_{\square} \cap \mathcal{L}=\emptyset$ and $\mathcal{L}_{\square} \subset \mathcal{L}_{M}$ (the set of all modal formulae, including nested ones). MEL is equipped with the following axioms:

1. $\phi \rightarrow(\psi \rightarrow \phi)$.

2. $(\psi \rightarrow(\phi \rightarrow \mu)) \rightarrow((\psi \rightarrow \phi) \rightarrow(\psi \rightarrow \mu))$.

3. $\left(\phi^{\prime} \rightarrow \psi^{\prime}\right) \rightarrow(\psi \rightarrow \phi)$

(K) $\square(p \rightarrow q) \rightarrow(\square p \rightarrow \square q)$.

(D) $\square \alpha \rightarrow \diamond \alpha$.

(N) if $\vdash_{P L} \alpha$ then $\square \alpha$.

and the inference rule is modus ponens. As usual, the possible modality $\diamond$ is defined as $\diamond \alpha \equiv\left(\square \alpha^{\prime}\right)^{\prime}$. The first three axioms are those of PL and the other those of modal logic KD. Axiom (N) is inspired from the necessitation rule that cannot be written in MEL. The following axioms (M) and (C) are implied by the above system:

$$
(M) \square(\alpha \wedge \beta) \rightarrow(\square \alpha \wedge \square \beta) ;
$$




$$
(C)(\square \alpha \wedge \square \beta) \rightarrow \square(\alpha \wedge \beta) \text {. }
$$

MEL is the subjective fragment of KD (or S5) without modality nesting.

The MEL semantics is very simple [12]. Let $\Omega$ be the set of $\mathcal{L}$ interpretations: $\{\omega: \mathcal{V} \rightarrow\{0,1\}\}$. The set of models of $\alpha$ is $[\alpha]=\{\omega: \omega \models \alpha\}$. A (meta)interpretation of $\mathcal{L}_{\square}$ is a non-empty set $E \subseteq \Omega$ of interpretations of $\mathcal{L}$ interpreted as an epistemic state. We define satisfiability as follows:

- $E \models \square \alpha$ if $E \subseteq[\alpha]$ ( $\alpha$ is certainly true in the epistemic state $E$ )

- $E \models \phi \wedge \psi$ if $E \models \phi$ and $E \models \psi$;

$-E=\phi^{\prime}$ if $E \models \phi$ is false.

MEL is sound and complete with respect to this semantics [13].

We remark that in this framework, uncertainty modeling is Boolean but possibilistic. The satisfiability $E \models \square \alpha$ can be written as $N([\alpha])=1$ in the sense of a necessity measure computed with the possibility distribution given by the characteristic function of $E$. Axioms (M) and (C) lay bare the connection with possibility theory [39], as they state the equivalence between $(\square \alpha \wedge \square \beta)$ and $\square(\alpha \wedge \beta)$.

We can justify the choice of this minimal modal formalism. It is the most simple logic to reason on incomplete propositional information. We only need to express that a proposition in PL is certainly true, certainly false or unknown as well as all the logical combinations of these assertions.

In $[24,26]$ we have proposed to translate three-valued logics of incomplete information into MEL, provided that the third truth-value refers to the idea of unknown Boolean truth-value. Let $a$ be a Boolean variable and $t(a)$ indicate the knowledge we have about $a$, that is:

- $\mathbf{1}$ certainly true, the Boolean value of $a$ is 1 ;

- $\mathbf{0}$ certainly false, the Boolean value of $a$ is 0 ;

$-\frac{1}{2}$ unknown, the Boolean value of $a$ is 0 or 1 .

For the sake of clarity, we have used different symbols $\mathbf{0}, \mathbf{1}, \frac{1}{2}$ for epistemic truthvalues with respect to ontic ones 0,1 . Under this understanding of the three epistemic truth-values, we can naturally translate three-valued truth-assigments to atomic propositions as follows:

$$
\begin{aligned}
& \mathcal{T}(t(a)=\mathbf{1})=\square a \\
& \mathcal{T}(t(a)=\mathbf{0})=\square a^{\prime} \\
& \mathcal{T}\left(t(a)=\frac{1}{2}\right)=\diamond a \wedge \diamond a^{\prime} \\
& \mathcal{T}\left(t(a) \geq \frac{1}{2}\right)=\diamond a \\
& \mathcal{T}\left(t(a) \leq \frac{1}{2}\right)=\diamond a^{\prime}
\end{aligned}
$$


Now, we want to map three-valued formulae to MEL formulae. Compound formulae are managed recursively. In the case of Łukasiewicz logic, we have:

$$
\begin{aligned}
\mathcal{T}(t(\alpha \sqcap \beta) \geq i) & =\mathcal{T}(t(\alpha) \geq i) \wedge \mathcal{T}(t(\beta) \geq i), i \geq \frac{1}{2} \\
\mathcal{T}(t(\alpha \sqcup \beta) \geq i) & =\mathcal{T}(t(\alpha) \geq i) \vee \mathcal{T}(t(\beta) \geq i), i \geq \frac{1}{2} \\
\mathcal{T}(t(\neg \alpha)=\mathbf{1}) & =\mathcal{T}(t(\alpha)=\mathbf{0})=\left(\mathcal{T}\left(t(\alpha) \geq \frac{1}{2}\right)\right)^{\prime} \\
\mathcal{T}\left(t(\neg \alpha) \geq \frac{1}{2}\right) & =\mathcal{T}\left(t(\alpha) \leq \frac{1}{2}\right)=(\mathcal{T}(t(\alpha)=\mathbf{1}))^{\prime} \\
\mathcal{T}\left(t\left(\alpha \rightarrow_{L} \beta\right)=\mathbf{1}\right) & =[\mathcal{T}(t(\alpha)=\mathbf{1}) \rightarrow \mathcal{T}(t(\beta)=\mathbf{1})] \\
& \wedge\left[\mathcal{T}\left(t(\alpha) \geq \frac{1}{2}\right) \rightarrow \mathcal{T}\left(t(\beta) \geq \frac{1}{2}\right)\right] \\
\mathcal{T}\left(t\left(\alpha \rightarrow_{L} \beta\right) \geq \frac{1}{2}\right) & =\mathcal{T}(t(\alpha)=\mathbf{1}) \rightarrow \mathcal{T}\left(t(\beta) \geq \frac{1}{2}\right)
\end{aligned}
$$

Note that even if we use the same symbols for connectives in L3 and MEL, we are moving from three-valued variables (formulae) to Boolean ones. In particular, in the case of atoms, we have

$$
\mathcal{T}\left(t\left(a \rightarrow_{L} b\right) \geq \frac{1}{2}\right)=(\square a)^{\prime} \vee \diamond b=\square a \rightarrow \diamond b
$$

and

$$
\begin{aligned}
\mathcal{T}\left(t\left(a \rightarrow_{L} b\right)=\mathbf{1}\right) & \left.=\left((\square a)^{\prime} \vee \square b\right) \wedge\left((\diamond a)^{\prime} \vee \diamond b\right)=\square a^{\prime} \vee \square b \vee\left((\square a)^{\prime} \wedge \diamond b\right)\right) \\
& =(\square a \rightarrow \square b) \wedge(\diamond a \rightarrow \diamond b) .
\end{aligned}
$$

It can be easily shown that by this translation, only a fragment of MEL can be captured by Łukasiewicz logic. Namely, $\mathcal{L}_{\square}^{\mathcal{L}}=\square a\left|\square a^{\prime}\right| \phi^{\prime}|\phi \vee \psi| \phi \wedge \psi$. That is, we can only have modalities in front of literals.

Finally, we note that this translation makes sense; that is tautologies are preserved by the translation and we can reason in three-valued logic inside MEL. More formally, the following two theorems hold [24,26]:

Theorem 5.1. If $\alpha$ is an axiom of Eukasiewicz (but also, Gödel, Nelson) logic, then $\mathcal{T}(t(\alpha))$ is a tautology in $M E L$.

Theorem 5.2. Let $\alpha$ be a formula in Eukasiewicz (but also, Nelson) logic $L_{3}$ and $B_{L}$ a knowledge base in this logic. Then, $B_{L} \vdash \alpha$ in $L_{3}$ iff $\mathcal{T}\left(B_{L}\right) \vdash \mathcal{T}(t(\alpha)=$ 1) in $M E L$.

Due to the equivalence between L3 and PRL, we can also translate PRL into MEL. Let us start from the connectives: $\vee, \wedge, \neg$ are the same as in Eukasiewicz logic, the only difference is the necessity which is not a primitive operator in L3 but can be derived as follows:

$$
\left.\left.\mathcal{T}\left(t\left(\mathbf{\square}_{\mu}\right)\right)=\mathbf{1}\right)=\mathcal{T}\left(t(\mathbf{\square} \mu) \geq \frac{1}{2}\right)\right)=\mathcal{T}\left(t\left(\neg\left(\mu \rightarrow_{L} \neg \mu\right)\right)=\mathbf{1}\right)=\mathcal{T}(t(\mu)=\mathbf{1})
$$

which on atoms corresponds to $\left.\left.\left.\mathcal{T}\left(t\left(\mathbf{\square}_{a}\right)=1\right)\right)=\mathcal{T}\left(t\left(\mathbf{\square}_{a}\right)\right) \geq \frac{1}{2}\right)\right)=\square a$.

Consequently, PRL implication (i.e., Gaines-Rescher $\rightarrow_{14}$ ) becomes in MEL:

$$
\mathcal{T}\left(t\left(\mu \rightarrow_{14} \nu\right)=1\right)=\mathcal{T}\left(t\left(\mu \rightarrow_{14} \nu\right) \geq \frac{1}{2}\right)=(\square \mu \rightarrow \square \nu) \wedge(\diamond \mu \rightarrow \diamond \nu)
$$


which, as expected corresponds to the translation of any residuated implication into MEL.

Due to the above results, in particular Theorem 5.1, it is also possible to see that most PRL axioms translate into MEL tautologies.

Corollary 5.1. If $\phi$ is an axiom of PRL logic (but for RL8 and RL9), then $\mathcal{T}(t(\phi)=1)$ is a tautology in $M E L$.

Axioms RL8 and RL9 which involve nested modalities, are not directly expressible in MEL logic. However, in PRL, $\square \mu$ and $\boldsymbol{\square} \boldsymbol{\square} \mu$ are equivalent to $\boldsymbol{\square} \mu$ so that they do not need to be translated.

It is interesting to comment on the difference between the two translations of PRL into S5 and into MEL:

- The translation of PRL into MEL yields the L3-fragment of MEL, and we know from $[24,26]$ that the two logics are equivalent. In particular, the MEL translation does not involve at all the logical rendering of the approximated set underlying the pair $(\boldsymbol{\square}, \alpha)$ in PRL. The lack of expressiveness of PRL with respect to S5 for rough sets is highlighted by the small fragment of S5 attained by the exact translation of PRL into MEL.

- The translation of PRL into S5 highlights a possible logical expression of the set approximated by the combination of approximation pairs, but this expression involves modalities, and does not refer to a set expressible in the pure propositional language $\mathcal{L}$ contained in the one of S5 (see equation (9)). So, even if this translation reaches a language richer than MEL, it carries over to the logical level the semantic difficulty of applying a truth-functional view on approximation pairs, as pointed out in the previous section.

\section{Conclusion}

The main lesson of this paper is that there is a gap between rough sets and three-valued calculi of approximation pairs regardless of the chosen rich enough algebraic setting, since there are several equivalent ones in three-valued logics. Whether we use Łukasiewicz 3-valued MV algebra, Nelson semi-simple algebras or the pre-rough setting, we can only imperfectly capture the modal logic of rough sets, that is, S5, even if the three-valued approaches can be embedded in the modal setting.

Our exploration of the 28 basic implications and conjunctions acting on orthopairs of sets show that there is no way to find binary connectives on orthopairs that would correspond exactly to the approximation pair enclosing some appropriate Boolean combination of the two sets approximated by each orthopair. This Boolean combination either does not exist, or must involve the chosen equivalence relation in some way, and then it is not even unique. This result is a generalisation of Bonikowski [16] old finding, systematized to all reasonable three-valued conjunctions, disjunctions and implications. It also echoes early remarks on the impossibility of representing rough sets by three-valued fuzzy sets put forward by Pawlak himself [60] and more recently by Yao [74]. 
Among the perspectives of this paper, one may point out the potential of the MEL language for representing and reasoning about information tables with missing values. Information tables are often encoded in a logical format using languages such as Datalog. In the case of missing values, the L3 fragment of MEL with modalities in front of literals could typically represent information about objects in intension, as an alternative to the use of Kleene logic proposed very early by Codd [27]. It would be of interest to reconsider proposals for defining rough sets under incomplete information in the light of this modal translation of three-valued logics.

\section{References}

1. Adams, E.: The Logic of Conditionals. D. Reidel, Dordrecht (1975)

2. Asenjo, F.G., Tamburino, J.: Logic of antinomies. Notre Dame journal of formal logic 16, 17-44 (1975)

3. Atanassov, K.: Intuitionistic Fuzzy Sets. Physica-Verlag, Heidelberg (1999)

4. Avron, A.: On an implication connective of RM. Notre Dame Journal of Formal Logic 27, 201-209 (1986)

5. Avron, A.: Natural 3-valued logics - characterization and proof theory. J. Symb. Log. 56(1), 276-294 (1991)

6. Avron, A., Konikowska, B.: Rough sets and 3-valued logics. Studia Logica 90, 69-92 (2008)

7. Baets, B.D., Fodor, J.C.: Residual operators of uninorms. Soft Computing 3, 89$100(1999)$

8. Balbiani, P., Vakarelov, D.: A modal logic for indiscernibility and complementarity in information systems. Fundam. Inform. 50(3-4), 243-263 (2002)

9. Banerjee, M.: Rough sets and 3-valued Lukasiewicz logic. Fundamenta Informaticae 31(3/4), 213-220 (1997)

10. Banerjee, M., Chakraborty, K.: Algebras from rough sets. In: Pal, S., Skowron, A., Polkowski, L. (eds.) Rough-Neural Computing, pp. 157-188. Springer-Verlag (2004)

11. Banerjee, M., Chakraborty, M.: Rough sets through algebraic logic. Fundamenta Informaticae 28, 211-221 (1996)

12. Banerjee, M., Dubois, D.: A simple modal logic for reasoning about revealed beliefs. In: Sossai, C., Chemello, G. (eds.) Proc. ECSQARU 2009, Verona, Italy, LNAI 5590. pp. 805-816. Springer-Verlag (2009)

13. Banerjee, M., Dubois, D.: A simple logic for reasoning about incomplete knowledge. International Journal of Approximate Reasoning (2013), in press

14. Belnap, N.D.: A useful four-valued logic. In: Dunn, J.M., Epstein, G. (eds.) Modern Uses of Multiple-Valued Logic, pp. 8-37. D. Reidel (1977)

15. Bochvar, D.A.: On a three-valued logical calculus and its application to the analysis of the paradoxes of the classical extended functional calculus. History and Philosophy of Logic 2, 87-112 (1981)

16. Bonikowski, Z.: A certain conception of the calculus of rough sets. Notre Dame journal of Formal Logic 33(3), 412-421 (1992)

17. Borowski, L. (ed.): Selected works of J. Eukasiewicz. North-Holland, Amsterdam (1970) 
18. Cattaneo, G., Ciucci, D.: Algebraic structures for rough sets. In: Dubois, D., Grzymala-Busse, J., Inuiguchi, M., Polkowski, L. (eds.) Fuzzy sets and Rough Sets, LNCS - Transactions on Rough Sets, vol. 3135, chap. 12, pp. 218-264. Springer (2004)

19. Cattaneo, G., Ciucci, D., Dubois, D.: Algebraic models of deviant modal operators based on de Morgan and Kleene lattices. Inf. Sci. 181(19), 4075-4100 (2011)

20. Chellas, B.F.: Modal Logic, An Introduction. Cambridge University Press, Cambridge, MA (1988)

21. Ciucci, D.: Orthopairs: A Simple and Widely Used Way to Model Uncertainty. Fundam. Inform. 108(3-4), 287-304 (2011)

22. Ciucci, D., Dubois, D.: Truth-Functionality, Rough Sets and Three-Valued Logics. In: Proceedings ISMVL. pp. 98-103 (2010)

23. Ciucci, D., Dubois, D.: Relationships between connectives in three-valued logics. In: Proc. IPMU Conference, Catania. CCIS, vol. 297, pp. 633-642. Springer (2012)

24. Ciucci, D., Dubois, D.: Three-valued logics for incomplete information and epistemic logic. In: Proc. 13th European Conference on Logics in Artificial Intelligence (JELIA), Toulouse, France (September 2012)

25. Ciucci, D., Dubois, D.: A map of dependencies among three-valued logics. Information Sciences 250, 162-177 (2013), Corrigendum: Information Sciences, 256,234-235 (2014)

26. Ciucci, D., Dubois, D.: A modal theorem-preserving translation of a class of threevalued logics of incomplete information. Journal of Applied Non-Classical Logics 23, 321-352 (2013)

27. Codd, E.F.: Extending the database relational model to capture more meaning. ACM Trans. Database Syst. 4(4), 397-434 (1979)

28. Couso, I., Dubois, D.: Rough sets, coverings and incomplete information. Fundamenta Informaticae 108(3-4), 223-247 (2011)

29. De Baets, B., Fodor, J.C., Ruiz-Aguilera, D., Torrens, J.: Idempotent uninorms on finite ordinal scales. International Journal of Uncertainty, Fuzziness and Knowledge-Based Systems pp. 1-14 (2009)

30. Dempster, A.P.: Upper and lower probabilities induced by a multivalued mapping. The Annals of Statistics 28, 325-339 (1967)

31. Deschrijver, G., Kerre, E.E.: On the relationship between some extensions of fuzzy set theory. Fuzzy Sets and Systems 133(2), 227-235 (2003)

32. D’Ottaviano, I.M.L., da Costa, N.C.A.: Sur un problème de Jaśkowski. Comptes Rendus de l'Académie des Sciences 270, 1349-1353 (1970)

33. Dubois, D.: Degrees of truth, ill-known sets and contradiction. In: BouchonMeunier, B., Magdalena, L., Ojeda-Aciego, M., Verdegay, J.L., Yager, R. (eds.) Foundations of Reasoning under Uncertainty, Studies in Fuzziness and Soft Computing, vol. 249, pp. 65-83. Springer (2010)

34. Dubois, D., Gottwald, S., Hájek, P., Kacprzyk, J., Prade, H.: Terminological difficulties in fuzzy set theory - the case of "intuitionistic fuzzy sets". Fuzzy Sets and Systems 156(3), 485-491 (2005)

35. Dubois, D., Prade, H.: Fuzzy-set-theoretic differences and inclusions and their use in the analysis of fuzzy equations. Control and Cybernetics 13(3), 129-146 (1984)

36. Dubois, D., Prade, H.: A theorem on implication functions defined from triangular norms. Stochastica VIII, 267-279 (1984)

37. Dubois, D., Prade, H.: Twofold fuzzy sets and rough sets -some issues in knowledge representation. Fuzzy sets and systems 23, 3-18 (1987) 
38. Dubois, D., Prade, H.: Conditional Objects as Nonmonotonic Consequence Relationships. IEEE Transaction of Sysyems, Man, and Cybernetics 24(12), 1724-1740 (1994)

39. Dubois, D., Prade, H.: Possibility theory, probability theory and multiple-valued logics: A clarification. Ann. Math. and AI 32, 35-66 (2001)

40. Dubois, D., Prade, H.: Gradualness, uncertainty and bipolarity: Making sense of fuzzy sets. Fuzzy Sets and Systems 192, 3-24 (2012)

41. Düntsch, I.: A logic for rough sets. Theoretical Computer Science 179, 427-436 (1997)

42. Fariñas del Cerro, L., Orlowska, E.: Dal - a logic for data analysis. Theor. Comput. Sci. 36, 251-264 (1985)

43. Fitting, M.: A Kripke-Kleene semantics for logic programs. J. Log. Program. 2(4), 295-312 (1985)

44. Gaines, B.R.: Foundations of fuzzy reasoning. Int. J. of Man-Machine Studies 6, 623-668 (1976)

45. Gehrke, M., Walker, E.: On the structure of Rough Sets. Bulletin Polish Academy of Science (Mathematics) 40, 235-245 (1992)

46. Gentilhomme, M.Y.: Les ensembles flous en linguistique. Cahiers de Linguistique Théorique et Appliquée, Bucarest 47, 47-65 (1968)

47. Gödel, K.: Zum intuitionistischen Aussagenkalkül. Anzeiger Akademie der Wissenschaften Wien 69, 65-66 (1932)

48. Hájek, P.: Metamathematics of Fuzzy Logic. Kluwer, Dordrecht (1998)

49. Iturrioz, L.: Rough sets and three valued structures. In: Orlowska, E. (ed.) Logic at work, pp. 596-603. Springer (1999)

50. Jaśkowski, S.: Propositional calculus for contradictory deductive systems. Studia Logica 24, 143-160 (1969)

51. Khan, M.A., Banerjee, M.: A logic for complete information systems. In: Sossai, C., Chemello, G. (eds.) Proc. ECSQARU 2009, Verona, Italy, LNAI 5590. pp. 829-840. Springer-Verlag (2009)

52. Kleene, S.C.: Introduction to Metamathematics. North-Holland Pub. Co., Amsterdam (1952)

53. Lawry, J., González Rodríguez, I.: A bipolar model of assertability and belief. Int. J. Approx. Reasoning 52(1), 76-91 (2011)

54. Mas, M., Mayor, G., Torrens, J.: t-Operators and Uninorms on a Finite Totally Ordered Set. International Journal of Intelligent Systems 14, 909-922 (1999)

55. Nelson, D.: Constructible Falsity. J. of Symbolic Logic 14, 16-26 (1949)

56. Orlowska, E.: A logic of indiscernibility relations. In: Computation Theory, pp. 177-186. No. 208 in Lecture Notes in Computer Sciences, Springer-Verlag, Berlin (1985)

57. Orlowska, E., Pawlak, Z.: Representation of nondeterministic information. Theor. Comput. Sci. 29, 27-39 (1984)

58. Pagliani, P.: Rough Sets and Nelson Algebras. Fundamenta Informaticae 27(2,3), 205-219 (1996)

59. Pagliani, P.: Rough set theory and logic-algebraic structures. In: Incomplete Information: Rough Set Analysis, pp. 109-190. Physica-Verlag, Heidelberg (1998)

60. Pawlak, Z.: Rough sets and fuzzy sets. Fuzzy Sets and Systems 17, 99-102 (1985)

61. Pawlak, Z., Skowron, A.: Rudiments of rough sets. Information Sciences 177, 3-27 (2007)

62. Pearce, D.: Equilibrium logic. Annals of Mathematics and Artificial Intelligence 47, 3-41 (2006) 
63. Priest, G.: The logic of paradox. The Journal of Philosophical Logic 8, 219-241 (1979)

64. Sen, J., Chakraborty, M.: A study of interconnections between rough and 3-valued Lukasiewicz logics. Fundam. Inform. 51, 311-324 (2002)

65. Sette, A.M.: On propositional calculus P_1. Math. Japon. 16, 173-180 (1973)

66. Shapiro, S.: Vagueness in Context. Oxford University Press (2006)

67. Smith, K.C.: The prospects for multivalued logic: A technology and applications view. IEEE Trans. Computers 30(9), 619-634 (1981)

68. Sobociński, B.: Axiomatization of a partial system of three-value calculus of propositions. J. of Computing Systems 1, 23-55 (1952)

69. Vakarelov, D.: A modal logic for similarity relations in Pawlak knowledge representation systems. Fundam. Inform. 15(1), 61-79 (1991)

70. Vakarelov, D.: Modal logics for knowledge representation systems. Theor. Comput. Sci. 90(2), 433-456 (1991)

71. Walker, E.A.: Stone algebras, conditional events, and three valued logic. IEEE Transaction of Systems, Man, and Cybernetics 24(12), 1699-1707 (1994)

72. Yao, Y.: Interval sets and interval-set algebras. In: Proceedings of the 8th IEEE International Conference on Cognitive Informatics. pp. 307-314 (2009)

73. Yao, Y.Y.: Interval-set algebra for qualitative knowledge representation. In: AbouRabia, O., Chang, C.K., Koczkodaj, W.W. (eds.) ICCI. pp. 370-374. IEEE Computer Society (1993)

74. Yao, Y.: Semantics of fuzzy sets in rough set theory. Transactions on Rough Sets pp. 297-318 (2004)

75. Yao, Y., Lin, T.: Generalization of rough sets using modal logic. Automation and Soft Computing 2(2), 103-120 (1996)

76. Yao, Y., Wang, S., Lin, T.: A review of rough set models. In: Polkowski, L., Skowron, A. (eds.) Rough Sets and Data Mining: Analysis for Imprecise Data, pp. 47-75. Kluwer Academic Publishers (1997)

77. Zadeh, L.A.: The concept of a linguistic variable and its application to approximate reasoning - part I. Information Sciences 8, 199-251 (1975) 